\title{
Relating Chain Conformation to the Density of States and Charge Transport in Conjugated Polymers: The Role of the $\beta$-phase in Poly(9,9-dioctylfluorene)
}

\author{
Xingyuan Shi (侍兴源), ${ }^{1, *}$ Vojtech Nádaždy, ${ }^{2}$ Aleksandr Perevedentsev, ${ }^{3,+}$ Jarvist M. Frost, ${ }^{1}$ Xuhua Wang, ${ }^{1}$ \\ Elizabeth von Hauff, ${ }^{4}$ Roderick C. I. MacKenzie, ${ }^{5}$ and Jenny Nelson ${ }^{1, \dagger}$ \\ ${ }^{1}$ Department of Physics and Centre for Plastic Electronics, Imperial College London, \\ South Kensington Campus, SW7 2AZ London, United Kingdom \\ ${ }^{2}$ Institute of Physics, Slovak Academy of Sciences, Dúbravská cesta 9, 84511 Bratislava, Slovakia \\ ${ }^{3}$ Department of Materials, Eidgenössische Technische Hochschule (ETH) Zürich, \\ Vladimir-Prelog-Weg 5, 8093 Zürich, Switzerland \\ ${ }^{4}$ Department of Physics and Astronomy, Vrije Universiteit Amsterdam, \\ De Boelelaan 1081, 1081 HV Amsterdam, The Netherlands \\ ${ }^{5}$ Faculty of Engineering, University of Nottingham, \\ University Park, NG7 2RD Nottingham, United Kingdom
}

(Received 4 August 2018; revised manuscript received 18 February 2019; published 24 May 2019)

\begin{abstract}
Charge transport in $\pi$-conjugated polymers is characterized by a strong degree of disorder in both the energy of conjugated segments and the electronic coupling between adjacent sites. This disorder arises from variations in the structure and conformation of molecular units, as well as the weak intermolecular binding interactions. Although disorder in molecular conformation can be expected to influence the density of states (DOS) distribution - and hence, optoelectronic properties of the material — until now, there has been no direct study of the relationship between a distinct conformational defect and the charge transport properties of a conjugated polymer. Here, we investigate the impact of introducing an extended, planarized chain geometry, known as the " $\beta$-phase," on hole transport through otherwise amorphous films of poly $(9,9$ dioctylfluorene) (PFO). We show that while $\beta$-phase introduces a striking drop of about a hundredfold in time-of-flight (TOF) hole mobility $\left(\mu_{h}\right)$ at room temperature, it reduces the steady-state $\mu_{h}$ measured from hole-only devices by a factor of less than about 5 . In order to reconcile these observations, we combine high-dynamic-range TOF photocurrent spectroscopy and energy-resolved electrochemical impedance spectroscopy to extract the hole DOS of the conjugated polymer. Both methods show that the effect of the $\beta$-phase content is to introduce a sharp sub-bandgap feature into the DOS of glassy PFO lying about $0.3 \mathrm{eV}$ above the highest occupied molecular orbital. The observed energy of the conformational trap is consistent with electronic structure calculations using a tight-binding approach. Using the obtained DOS with a driftdiffusion model capable of resolving charge carriers in both time and energy, we show how the seemingly contradictory transport phenomena obtained via the time-resolved, frequency-resolved, and steady-state methods are reconciled. The results highlight the significance of energetic redistribution of charge carriers in affecting transport behavior. This work demonstrates how charge-carrier mobility in organic semiconductors can be controlled via molecular conformation, and it resolves a long-standing debate over how different (equilibrium versus nonequilibrium) transport techniques reveal electronic properties of disordered solids in a unified manner.
\end{abstract}

DOI: 10.1103/PhysRevX.9.021038

\footnotetext{
* Corresponding author. x.shi12@imperial.ac.uk

Corresponding author. jenny.nelson@imperial.ac.uk

${ }^{\star}$ Present address: Institut de Ciència de Materials de Barcelona (ICMAB-CSIC), Campus de la UAB, 08193 Bellaterra, Spain.

Published by the American Physical Society under the terms of the Creative Commons Attribution 4.0 International license. Further distribution of this work must maintain attribution to the author(s) and the published article's title, journal citation, and DOI.
}

Subject Areas: Chemical Physics, Materials Science, Semiconductor Physics

\section{INTRODUCTION}

Dating from early research into amorphous inorganic semiconductors [1-5] to recent studies on $\pi$-conjugated molecular semiconducting materials [6-13], there has been consistent interest in understanding the impact of localized, sub-bandgap states on charge transport in disordered electronic materials. Organic semiconductors are particularly interesting on account of the strong interaction between the electronic states and vibrational modes of the materials, which makes energetic disorder almost 
(a)

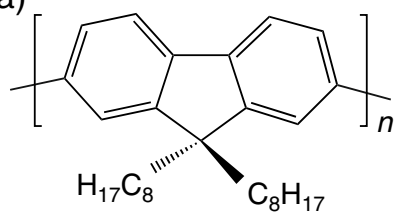

(b)

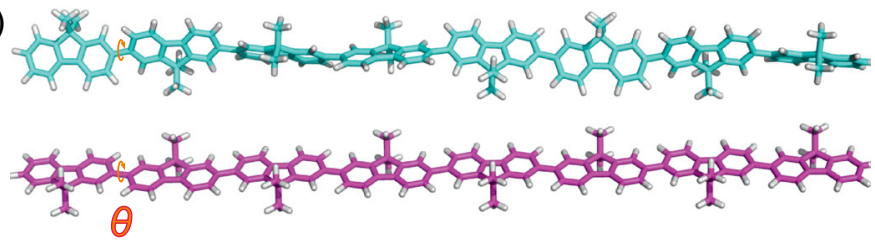

FIG. 1. (a) The chemical structure of PFO. Each of the di- $n$-octyl side chains attached to the central carbon atom $\left(\mathrm{C}_{9}\right)$ of a monomer is abbreviated as $\mathrm{C}_{8} \mathrm{H}_{17}$. (b) Molecular geometries of an oligofluorene (side chains represented by methyl groups for clarity) in the minimum potential energy, glassy conformation with dihedral angles $\theta$ of about $45^{\circ}$ or $135^{\circ}$ (top, cyan) and in the $\beta$-phase conformation, with $\theta \approx 180^{\circ}$ (bottom, magenta).

unavoidable and hard to control. A variety of photoelectron, optical, and capacitance spectroscopies has been exploited to gain information about electronic structure of organic semiconductors. Techniques including ultraviolet or x-ray photoelectron spectroscopy (UPS or XPS) [14,15], photoelectron spectroscopy in air (PESA) [16,17], and photothermal deflection spectroscopy (PDS) [18,19], as well as capacitance methods such as deep-level transient spectroscopy (DLTS) [20,21] and capacitance-frequency or voltage [22-25] reveal and quantify the presence of localized states lying within the bandgap of such materials. In order to correlate the distribution of sub-bandgap density of states (DOS) with its impact on charge transport, a variety of time-resolved, frequency-resolved, and temperaturedependent (opto)electrical probes has been used to extract information about the sub-bandgap DOS and its relation to charge transport. For instance, transient current measurements including time-of-flight (TOF) photocurrent may be interpreted in terms of the rate of charge-carrier release from trap states [2-4,26,27], thermally stimulated current (TSC) [28-31] or luminescence (TSL) [32,33] measurements analyzed in terms of thermal activation out of trap states, and frequency-domain methods [34-40] interpreted in terms of electrical perturbation of charge induced by a frequency-variable small-signal ac voltage superimposed on a dc bias, such that carrier motion as well as trapping and detrapping processes can be observed. However, these varying experimental methods generally expose devices to different measurement conditions (e.g., applied bias, device thickness, choice of contacts, use of background illumination or laser pulse), and data interpretation involves different theoretical approximations. There is as yet no consensus on how to relate charge transport phenomena-including charge-carrier mobility, field dependence of mobility, and degree of carrier transport dispersion - to the sub-bandgap DOS using experimental methods. The challenge of relating transport properties to these localized states is complicated by the fact that (i) different methods can deliver different values of charge-carrier mobility even for the same material systems processed via identical protocols and that (ii) the particular origin and form of the sub-bandgap DOS in a disordered semiconductor is not normally uniquely known. Lack of knowledge of the DOS makes it impossible to predict the charge transport behavior using any transport model that is capable of accounting for the presence of energetic disorder and/or trap states.

In order to address these challenges, materials systems are needed where the sub-bandgap DOS is well defined and tunable in a controlled manner. In the case of organic semiconductors, variations in the energy of electronic states may arise from effects including chemical defects, impurities, supramolecular structural variations and polymorphs, dynamic disorder due to thermal fluctuations, or intermolecular electrostatic and polarization interactions [8,41-44]. In general, the exact origin of trap states at any given depth is not known. It is typical for the sub-bandgap DOS profile to appear exponential over a range of energies $[9,11,45]$ although without any clear physical reason. In the case of conjugated polymers, one important source of site energy distribution is conformational disorder due to variation in intrachain torsion and the resulting variations in the extent of $\pi$-conjugation along the polymer backbone. Several experimental [17,46-52] and modeling [42,53-59] studies have addressed the impact of molecular conformation on electronic structure and transport properties. However, despite sound models for the dependence of electronic structure on conformation, the large conformational phase space of conjugated polymers has made it difficult to relate the DOS and, ultimately, the recorded electronic transport properties explicitly to the presence of any conformer, even when certain conformers are deliberately introduced $[47,60]$.

In the present work, we select the conjugated polymer, poly(9,9-dioctylfluorene) (PFO), for detailed study. PFO expresses several polymorphs, one of which, termed the $\beta$-phase (see Fig. 1), features an extended, planar-zigzag chain conformation [61-63] that can be reproducibly introduced into amorphous (or "glassy") phase PFO layers via exposure to solvent vapors $[63,64]$, and whose presence can readily be identified spectroscopically via its UV-Vis absorption, photoluminescence [63,65], and Raman scattering [66,67] "fingerprints." Moreover, the hole mobility $\left(\mu_{h}\right)$ in PFO is reported to be a strong function of the phase of the polymer $[68,69]$, and recent studies $[70,71]$ showed that glassy PFO exhibits a room-temperature (RT) time-offlight (TOF) $\mu_{h}$ that is apparently 2 orders of magnitude higher than that of the same medium contaminated with $\beta$-phase. 
We show how the DOS of the polymer can be determined using two independent methods, namely, high-dynamicrange (HDR) TOF photocurrent measurements and energy-resolved electrochemical impedance spectroscopy (ER-EIS), and that the impact of introducing $\beta$-phase into the polymer is to induce a sharp sub-bandgap feature in the DOS some $0.3 \mathrm{eV}$ above the highest occupied molecular orbital (HOMO) of the glassy polymer. We show that the effect of the $\beta$-phase conformer on different charge transport measurements (including transient, frequencyresolved, and steady-state) can be explained quantitatively in terms of the DOS due to this conformational defect. Furthermore, we demonstrate how apparent differences in transport data from the complementary set of (opto)electrical probes can be resolved using a time- and energyresolved device model [11] that allows for the energetic redistribution of charge carriers.

This work provides a first demonstration that knowledge of a particular conformational defect can be related quantitatively to the effect on transport measurements, meaning that the effect of conformational defects on charge transport properties of a conjugated polymer can, in principle, be predicted. This enables the charge transport properties of conformationally disordered polymer materials to be modeled. In addition, the explanation of apparently different observations of transport in terms of a single picture of the density of states shows how, when appropriate models of charge transport are used, different measurement methods can be reconciled. This finding resolves a long-standing controversy over the ability of different methods to faithfully probe the charge transport properties of a disordered solid.

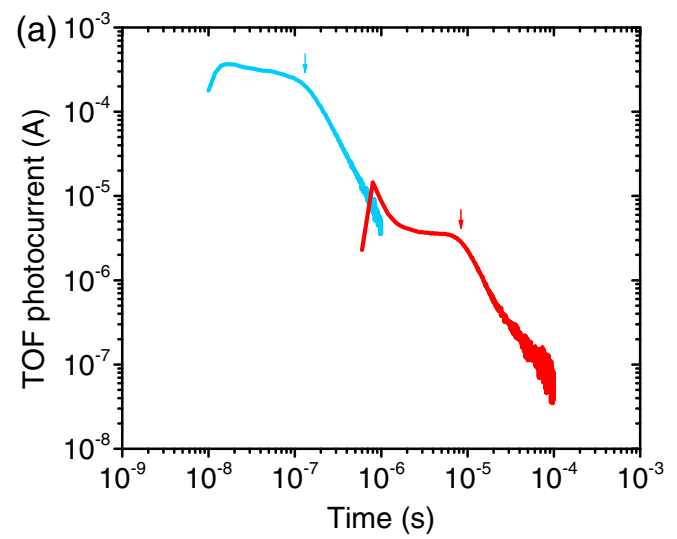

\section{IMPACT OF THE $\beta$-PHASE ON HOLE TRANSPORT IN PFO}

\section{A. Temperature-dependent time-of-flight charge-carrier mobility}

TOF hole mobility measurements on optically thick PFO devices prepared in the two different forms (glassy and $\beta$ phase embedded) reveal a clear trend in mobility with microstructure. Figure 2(a) shows double-logarithmic plots of the transient hole photocurrent measured at RT (about $290 \mathrm{~K}$ ) for about $1.8-\mu \mathrm{m}$-thick PFO films sandwiched between hole-blocking indium tin oxide (ITO) and aluminium (Al) electrodes under an applied bias of $10 \mathrm{~V}$ (i.e., at an electric field of about $5.6 \times 10^{4} \mathrm{~V} \mathrm{~cm}^{-1}$ ). Such identical conditions mean that transient hole transport for the different devices can be directly compared from the carrier transit times $t_{\text {tr }}$ defined here as the intersection of the asymptotes to the current plateau and the trailing edge of the transient in the double-logarithmic plot. A reduction of about a hundredfold in TOF hole mobility upon generating $\beta$ phase into fully glassy layers is evident. Figure 2(b) presents TOF $\mu_{h}$ measured as a function of temperature and electric field in devices of structure ITO/PFO $\sim 1.8 \mu \mathrm{m} / \mathrm{Al}$, under $355-\mathrm{nm}$ pulsed laser excitation delivered through the ITO front contact (see the Appendix A for molecular-weight properties of the two PFO batches used, Appendix B for processing details of PFO samples and devices, and Appendix C, Part 1 for the TOF experimental setup). Substantially higher TOF $\mu_{h}$ were obtained for the pristine glassy PFO devices $\left(\sim 3 \times 10^{-2} \mathrm{~cm}^{2} \mathrm{~V}^{-1} \mathrm{~s}^{-1}\right.$ at RT) than the devices in which $\beta$-phase had been introduced (referred to as " $\beta$-embedded"

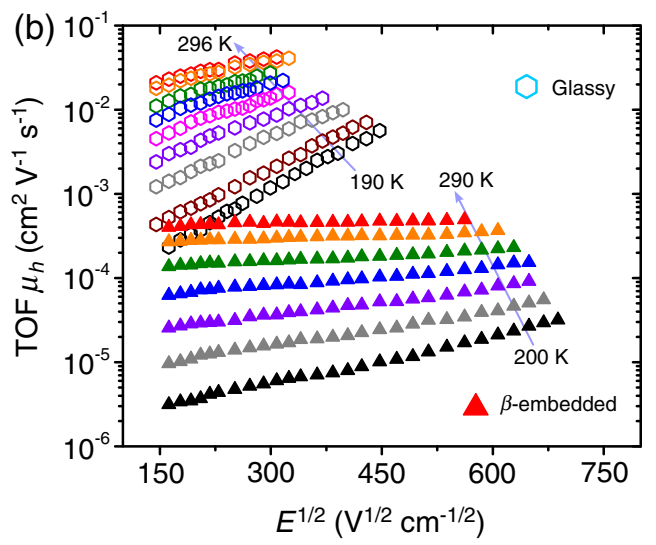

FIG. 2. (a) Representative TOF photocurrent transients for glassy (blue line) and $\beta$-phase embedded (red line) HMW PFO devices. All films had very comparable thickness $(\sim 1.8 \mu \mathrm{m})$, and measurements were performed under identical conditions $\left(V_{\text {bias }}=10 \mathrm{~V}\right.$, i.e., at $E \approx 5.6 \times 10^{4} \mathrm{~V} \mathrm{~cm}^{-1}$; and $T=290 \mathrm{~K}$ ). For each case, the carrier transit time $t_{\text {tr }}$ (indicated by an arrow) was extracted by locating the point of intersection of two tangents (omitted for clarity) fitted to the pre- and post-transit regimes of the transient on the doublelogarithmic scale. The variation in onset of the initial spike reflects the different choices of the temporal resolution or window to best capture the photocurrent transients. No manual offset was applied to the transients, and the photocurrent levels are absolute. (b) PooleFrenkel plots of TOF hole mobility $\left(\mu_{h}\right)$ as a function of the square root of applied electric field, measured at different temperatures for HMW TOF devices made with pristine glassy PFO (top set, open hexagons) and with PFO containing $\beta$-phase structural minority introduced by exposure to toluene vapor for an hour (bottom set, filled triangles). Gaussian disorder model analysis of these data is presented in Appendix F. The temperatures measured were-in order of increasing $\mu_{h}-190,200,215,230,245,260,270,290$, and $296 \mathrm{~K}$ for the glassy set; and 200, 215, 230, 245, 260, 275, and $290 \mathrm{~K}$ for the $\beta$-phase embedded set. 
devices; $\sim 4 \times 10^{-4} \mathrm{~cm}^{2} \mathrm{~V}^{-1} \mathrm{~s}^{-1}$ at RT). UV-Vis absorption and photoluminescence measurements were carried out to ensure that the chosen processing routes yielded PFO films in the required microstructures (see Appendix D). Each TOF $\mu_{h}$ value in Fig. 2(b) was estimated by ascribing a transit time to the point of intersection of two tangents fitted to the short-time ("pretransit") and long-time ("posttransit") parts of each photocurrent transient on the double-logarithmic scale, thus marking the so-called "knee" position [see Fig. 2(a) for representative TOF transients]. The TOF $\mu_{h}$ obtained in this way is reduced by around 2 orders of magnitude through the introduction of only about $4.6 \pm 0.4 \mathrm{vol} \%$ of $\beta$-phase (as estimated in Appendix E). This observation is in accordance with the impact of $\beta$-phase on TOF $\mu_{h}$ reported in Ref. [70]. The magnitude of this effect is dependent upon the molecularweight properties of the polymer used, with the lowmolecular-weight (LMW) material leading to a larger fraction ( $~ 6 \pm 0.1 \mathrm{vol} \%)$ of $\beta$-phase dispersed in the PFO film and a slightly larger impact on the reduction of $\mu_{h}$ [see the Supplemental Material (SM) [72], Sec. III, Fig. S8(b)]; for clarity, we present all hole transport data on LMW PFO in the SM [72], whereas only high-molecular-weight (HMW) results are included in the main text). Figure 2(b) also indicates that the sensitivity of TOF $\mu_{h}$ to electric field is greater for the glassy, high-mobility device. Previously, different field dependences have been assigned to the degree of configurational disorder [6] that leads to differences in the spread of electronic coupling between adjacent localized electronic states. However, such a model would only be applicable to relatively homogeneous and isotropic media rather than structurally heterogeneous systems as in the case of the $\beta$-phase embedded films studied here.

To characterize the temperature dependence of the fielddependent TOF $\mu_{h}$ and enable comparison with previously published data on polyfluorene-based materials, we have analyzed the data using the Gaussian disorder model (GDM) [6]. This analysis (see Appendix F) provides a higher value for the energetic disorder $\sigma_{\mathrm{GDM}}$-defined as the standard deviation (or breadth) of a Gaussian distribution of (spatially uncorrelated) site energies-for the $\beta$-phase embedded device $\left(\sigma_{\mathrm{GDM}, \beta \text {-embedded }} \approx 85 \mathrm{meV}\right)$ than for the glassy device $\left(\sigma_{\mathrm{GDM}, \text { glassy }} \approx 75 \mathrm{meV}\right)$. It also yields a hightemperature limit of TOF $\mu_{h}\left(\mu_{h 0(T \rightarrow \infty)}\right)$ for the pristine glassy device of about $0.6 \mathrm{~cm}^{2} \mathrm{~V}^{-1} \mathrm{~s}^{-1}$, which is referred to, in the framework of the GDM, as the effective free-hole mobility through glassy phase PFO (for further clarification, see Appendix F). We use this $\mu_{h 0(T \rightarrow \infty)}$ value as an initial input for numerical device modeling (see the SM [72], Sec. I, Part B) and note that its magnitude is compatible with experimental estimates (on the order of about $1 \mathrm{~cm}^{2} \mathrm{~V}^{-1} \mathrm{~s}^{-1}$ ) for the ac intrachain mobility along randomly coiled PFO chains in dilute solutions $[52,73]$.

\section{B. Steady-state $J-V$ response from glassy and $\beta$-phase embedded PFO hole-only devices}

In order to test the consistency of our observed effect of $\beta$-phase on hole transport in PFO, we study the same systems with a second, steady-state technique. Steady-state dark current density-voltage $(J-V$; sometimes referred to as space-charge-limited current) measurements probe hole transport in a unipolar device structure with at least one Ohmic hole-injecting contact. Ultrathin (about 5-10 nm) $\mathrm{MoO}_{x}$ interlayers prior to top-contact metallization were previously shown to achieve Ohmic hole injection into the very deep-lying $\mathrm{HOMO}$ of $\mathrm{PFO}$, a level that is otherwise inaccessible via conductive polymer poly(3,4-ethylene dioxythiophene):poly(styrene sulfonate) (PEDOT:PSS) [74].
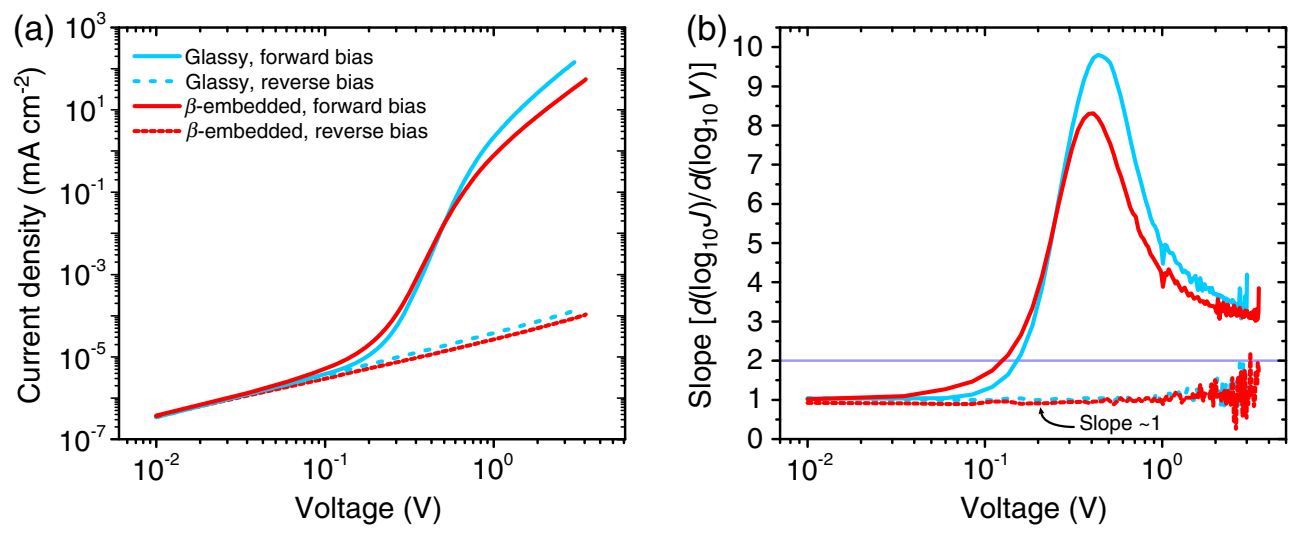

FIG. 3. (a) Double-logarithmic steady-state current density-voltage $(J-V)$ plots for devices of structure ITO/PEDOT:PSS/PFO $(\sim 135 \mathrm{~nm}) / \mathrm{MoO}_{x} / \mathrm{Au}$ made with glassy (blue curves) and $\beta$-phase embedded (red curves) layers. The measurements were taken at $295 \mathrm{~K}$ in the dark. Forward-bias response is illustrated by solid lines and refers to hole injection through the $\mathrm{Au} / \mathrm{MoO}_{x} \mathrm{Ohmic}$ contact. Reverse-bias response (dashed lines) refers to hole injection through the ITO/PEDOT:PSS blocking contact. (b) Plots of the corresponding slopes of all dual-logarithmic-scale traces shown in subplot (a) against the logarithm of voltage [slope $\left.=d\left(\log _{10} J\right) / d\left(\log _{10} V\right)\right]$. The purple line at a slope of 2 marks the behavior of trap- and barrier-free space-charge-limited conduction at high bias, which is predicted by the Mott-Gurney theory. 
Figure 3(a) shows steady-state $J-V$ curves for hole-only devices of asymmetric structure ITO/PEDOT:PSS/PFO $(\sim 135 \mathrm{~nm}) / \mathrm{MoO}_{x} / \mathrm{Au}$, prepared with glassy and $\beta$-phase embedded PFO layers. Both devices exhibit clear current rectification behavior. The distinction between the steadystate current density levels in the high-bias, drift-dominant regime when holes are injected from the $\mathrm{Au} / \mathrm{MoO}_{x}$ contact (i.e., forward bias) suggests that there is a systematic difference in $\mathrm{dc} \mu_{h}$ between the glassy and $\beta$-phase embedded layers. However, it is a factor of about 2-4 rather than the 2-orders-of-magnitude difference probed by TOF. Here, we do not attempt to obtain mobility values directly from these curves since it has been previously shown $[75,76]$ that the mobility extracted from dark $J-V$ data by the commonly adopted Mott-Gurney law [77] is not reliable when—as in the present case - both a significant distribution of electronic traps and contact asymmetry (hence a built-in potential, $V_{\mathrm{bi}}$ ) are present in the devices.

\section{Mobility extracted from hole-only devices using impedance spectroscopy}

An alternative route to determining $\mathrm{dc} \mu_{h}$ in single-carrier devices, as put forward by Tripathi et al. [37], Martens et al. [78], and Tsang et al. [34], is to examine the frequencyresolved impedance or admittance response. Both techniques are able to provide insights into the degree of transport dispersion from the spectra obtained. Figure 4 is obtained under forward bias by recording the frequencydependent imaginary component of the impedance spectra [i.e., $-\operatorname{Im}(Z)$ versus frequency; see Appendix C, Part 4 and Appendix G, Part 1] for the same glassy and $\beta$-phase embedded hole-only devices as in Part B. The data show that the mildly field-dependent glassy-state dc $\mu_{h}$ of around $6 \times 10^{-5} \mathrm{~cm}^{2} \mathrm{~V}^{-1} \mathrm{~s}^{-1}$ to $1 \times 10^{-4} \mathrm{~cm}^{2} \mathrm{~V}^{-1} \mathrm{~s}^{-1}$ drops by a factor of about 2-5 upon inclusion of $\beta$-phase conformers. As with the steady-state dark $J-V$ response, $\beta$-phase reduces $\mu_{h}$, and the effect is again relatively moderate in comparison with the TOF results. Interestingly, the same set of impedance measurements on the LMW batch PFO systematically indicate a more pronounced impact of $\beta$ phase on hole transport, particularly in lowering the degree of transport dispersion-a finding that is consistent with the time-resolved photocurrent transients (see the SM [72], Sec. III, for details).

The value for the hole-only device mobility estimated from impedance spectroscopy in the $\beta$-phase embedded case agrees reasonably well with a prior report by Nicolai et al. [74] of $1.3 \times 10^{-5} \mathrm{~cm}^{2} \mathrm{~V}^{-1} \mathrm{~s}^{-1}$, based on analysis of dark $J-V$ measurements on room-temperature spincoated PFO films. We expect films prepared as described in that report to contain $\beta$-phase according to such film deposition protocol, although not explicitly stated by the authors, since prolonged solvent evaporation during the deposition process promotes chain relaxation during solidification and hence the formation of $\beta$-phase [79].

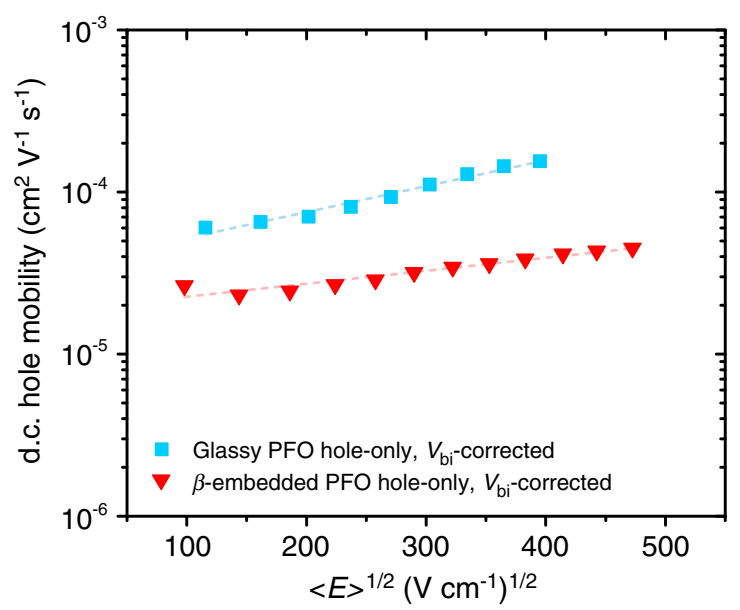

FIG. 4. Steady-state $\mu_{h}$ estimated from frequency-dependent impedance spectroscopy, as explained in Appendix C, Part 4 and Appendix G, Part 1, for hole injection through the $\mathrm{Au} / \mathrm{MoO}_{x}$ of the same devices used for steady-state $J-V$ measurements. The $\mu_{h}$ values are plotted as a function of the square root of the applied electric field. Hole mobilities were obtained by correcting for a built-in potential of about $0.47 \mathrm{~V}$ estimated from the capacitancevoltage $(C-V)$ response of the $\beta$-phase embedded device, and by using a dispersion factor of 0.5 (see Appendix C, Part 4 for details). The arithmetic means of electric field are calculated by deducting this built-in potential and averaging the effective applied bias across the layer thickness [see Appendix G, Part 2 for the estimation of $V_{\mathrm{bi}}$ using $C-V$ measurements on the hole-only devices, and Fig. 12(d) for a simulated set of electrostatic profiles across the active layers of the hole-only devices].

In summary, $J-V$ and impedance spectroscopy measurements on hole-only devices reveal that glassy PFO exhibits a higher steady-state hole mobility than $\beta$-phase embedded PFO by a factor of about 2-5. In contrast, TOF suggests the mobility of glassy PFO is around a hundredfold higher than that of $\beta$-phase embedded PFO. As we show below, our analysis suggests that a small fraction of $\beta$-phase chains dispersed within amorphous PFO should introduce trap states that penalize the hole mobility. However, the influence of these trap states on transport, in practice, appears to be very different depending upon which experimental technique is used. We also note a molecular-weight dependence of the impact of the induced $\beta$-phase fraction on mediating hole transport. To resolve the apparently contradictory observations, we next address the forms of the hole DOS in glassy PFO in the absence or presence of $\beta$-phase.

\section{PROBING THE HOLE DOS FOR GLASSY AND $\beta$-PHASE EMBEDDED PFO}

\section{A. Extracting DOS from high-dynamic-range time-of-flight measurements}

To probe the hole DOS in PFO, we first adapt the TOF technique where the dynamic range for photocurrent transients is extended to span around 7 to 8 orders of 
magnitude and, in turn, 6 orders in time up to about $10 \mathrm{~ms}$ (see Appendix C, Part 2 for method details). Such highdynamic-range measurements allow the DOS to be probed over several tenths of an electronvolt (about $0.5-0.55 \mathrm{eV}$ in the present study) relative to the transport level of charge carriers, provided that the internal field is sufficiently high and carrier retrapping can be neglected. The rationale is outlined as follows.

Transient current measurements are one of the most widely used means to relate transport to DOS. Based on the multiple-trapping model, Tiedje and Rose [2], and Orenstein and Kastner [3] related dispersive current transients, commonly observed in TOF photocurrent measurements, to the thermal release of trapped carriers out of a distribution of localized states as time elapses. Seynhaeve et al. extended the theory to estimate the band-tail distribution of hydrogenated amorphous silicon (a-Si:H) from experimental TOF data [4]. Such a model was later adapted by others $[9,11,80]$ to probe the DOS in organic bulkheterojunction photovoltaic devices. In this treatment, the time-dependent photocurrent $i(t)$ is determined by the thermally activated release of charge carriers from trap states, whose DOS distribution is given by $g\left(E^{*}\right)$. By mapping trap depth $E^{*}$ onto release time $t$, we obtain $g\left(E^{*}\right)$ from the photocurrent,

$$
g\left(E^{*}\right)=\frac{i(t) t}{q f_{\mathrm{loc}} V k_{\mathrm{B}} T},
$$

where $q$ is the elementary charge, $f_{\text {loc }}$ the fraction of localized states occupied immediately after the laser excitation, $V$ the device volume, and $k_{\mathrm{B}} T$ the thermal energy; $E^{*}$ represents the demarcation depth-relative to the transport level—of the energy level up to which the DOS is filled at time $t$ with negligible detrapping events. In deriving Eq. (1), it is assumed that the rate of carrier release is controlled by thermal activation; hence, one can map the time at which a carrier is released from a trap state onto the depth at which the carrier was detained as

$$
E^{*}(t)=k_{\mathrm{B}} T \ln \left(\nu_{0} t\right)
$$

where $\nu_{0}$ is the attempt-to-escape frequency. Note that the logarithmic dependence of energy on time means that a HDR photocurrent is needed to probe localized states deep in the gap. It should be stressed that the approximation to the DOS delivered from Eq. (1) only holds in the long-time limit (the so-called "post-transit regime"), where the TOF photocurrent is dominated by the detrapping of carriers via thermal activation $[2,4,80]$. It is assumed that, provided the internal field is high enough, retrapping would be minimal in this regime and that carrier loss by recombination is negligible (which is fair since TOF investigates unipolar transport) and the $f_{\text {loc }}$ stays frozen at site energies greater than the time-evolving demarcation energy $E^{*}$. Since the magnitude of $f_{\text {loc }}$ is generally not known, Eq. (1) delivers a relative DOS distribution. It is also assumed that the subbandgap DOS distribution remains unchanged over the temperature range probed and that trap states are empty prior to laser excitation due to ultralow dc dark current level $(<25 \mathrm{pA})$ recorded for the TOF devices within the bias range applied. In this study, we apply the HDR TOF method to probe the distribution of hole-trap states in PFO.

Figure 5(a) shows HDR hole transients from HMW PFO TOF devices prepared in the glassy state with and without the $\beta$-phase conformational heterogeneity introduced. To convert $i(t)$ to a DOS with an energy scale determined relative to the transport level [Eq. (2)], we must fix a value for the attempt-to-escape frequency $\nu_{0}$. Note that the choice of $\nu_{0}$ affects only the absolute position of the DOS on the energy scale but not the relative position or shape. We estimate $\nu_{0} \approx 10^{11} \mathrm{~Hz}$ from temperature-dependent HDR transient measurements by adjusting $\nu_{0}$ until particular features in the post-transit half of the transient curves coincide when plotted against energy [see vertical dashed lines in Fig. 5(a), downwards arrows in Fig. 5(b), and the figure caption for details]. We further show, in the SM [72], Sec. II, Part C, that an estimate of $\nu_{0}$ on the same order could be derived using an alternative analysis. Following Eq. (2), this value of $\nu_{0}$ translates the dynamic range of our HDR transients to a sub-bandgap depth of about 0.5$0.55 \mathrm{eV}$. The DOS curves mapped out in this way are plotted in Fig. 5(b). Figure 5(b) also shows that the shape of the glassy-state DOS extracted from the post-transit regime photocurrent is not dependent on the external bias voltage, suggesting that recombination does not affect the shape of the mapped DOS for the range of voltages studied and that carrier redistribution into deep localized states due to retrapping events is negligible. Furthermore, the temperature independence of the DOS confirms that no thermally induced microscopic phase transition takes place within the photoconductive layer in the narrow temperature range over which the measurements can be compared. (Extracted DOS can be compared only over a narrow temperature range because the upper limit is fixed by the need to avoid phase transitions near the glass transition temperature of the polymer, $T_{g} \sim 53{ }^{\circ} \mathrm{C}$ [71], and hence low-temperature measurements were performed in cryogenic conditions.) The energy-space map of the set of transients can thus be considered a realistic approximation to the DOS, and it supports the idea that we are working in a regime where Eq. (2) is valid.

Comparing the mapped hole DOS for glassy and $\beta$-phase embedded devices in Fig. 5(b), we can see that while both devices show a similar, approximately exponential tail of states at high depth (>ca. $0.42 \mathrm{eV})$, the $\beta$ phase embedded device alone exhibits an additional sharp feature at about $0.3 \mathrm{eV}$ above the transport level (direction referred according to electron energy). An exponential tail of states, where $g(E)$ varies according to $g(E) \propto \exp$ 

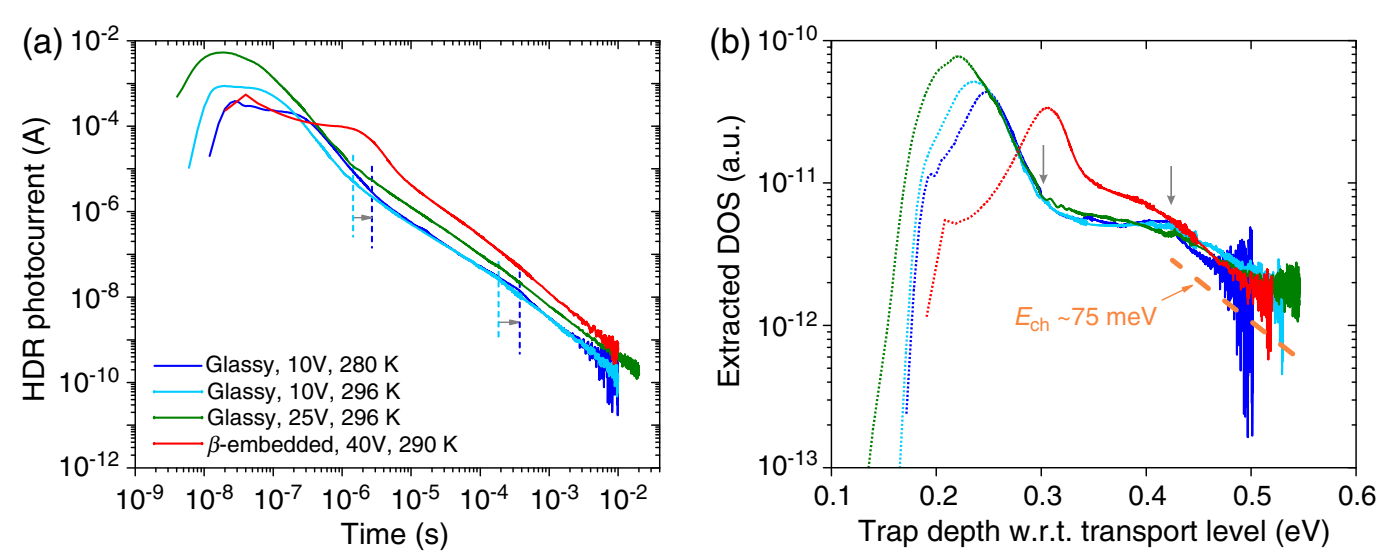

FIG. 5. (a) HDR TOF transients for a glassy HMW PFO device measured at different voltages and temperatures, in comparison with a transient for a $\beta$-phase embedded HMW PFO device acquired at a high bias. (b) Density of states extracted from the HDR transients in (a) using Eqs. (1) and (2) and an attempt-to-escape frequency $\nu_{0}$ of $1 \times 10^{11} \mathrm{~s}^{-1}$. The slope of the deep (>ca. $\left.0.42 \mathrm{eV}\right)$ tail of the extracted DOS for glassy PFO at around $75 \mathrm{meV}$ appears to be insensitive to measurement voltage and temperature, suggesting that the obtained representation of the DOS is robust. The DOS for the $\beta$-phase embedded device shows a peak at about $0.3 \mathrm{eV}$ above the transport edge, which is absent for the glassy device. The solid segments of each of the curves represent the range of energies over which the transient is in the post-transit regime, and the method of DOS extraction should be valid. To estimate $\nu_{0}$, we inspect TOF traces in subplot (a) collected from the glassy device under a controlled external bias of $10 \mathrm{~V}$, however differing by temperatures, at 296 (sky blue) and $280 \mathrm{~K}$ [(a "colder") royal blue]. Upon lowering the measurement temperature, it is evident that (i) the temporal position of $t_{\text {tr }}$ gets delayed as carrier transport slows down; (ii) the inflection features in the post-transit regime also get delayed — these are marked by dashed lines with a color scheme coherent with the respective traces. The trap-depth-dependent delay intervals are represented by the grey arrows; (iii) despite this systematic temporal shift, comparison of the two traces suggests a near independence of post-transit regime shape against variation of temperatures, within the range of which no microscopic phase transition is expected to occur for PFO, and hence no changes in the DOS distribution, as shown in subplot (b) with the DOS inflection features (marked by the grey downwards arrows) coinciding by adjusting the $\nu_{0}=1 \times 10^{11} \mathrm{~s}^{-1}$. We also note that the low-level (i.e., approximately sub-nA) photocurrent at $280 \mathrm{~K}$ suffers from an elevated noise floor due to the use of a cryogenic setup that unavoidably employs lengthy miniature coaxial cables for electrical signaling.

$\left(E / E_{\mathrm{ch}}\right)$, with $E_{\mathrm{ch}}$ being the characteristic energy of the tail of states, has been inferred from various attempts to measure the DOS of a conjugated polymer $[9,11,45,80]$. Such a $g(E)$ profile is consistent with a conformationally disordered and entangled polymer [56]. The characteristic energy observed here $\left(E_{\mathrm{ch}} \sim 75 \pm 5 \mathrm{meV}\right)$ is similar to values reported for other conjugated polymers. The clear extra peak or cliff that is visible only in the case of the $\beta$-phase embedded device suggests the presence of an additional, relatively welldefined distribution of defect states.

\section{B. Thin-film DOS mapping using energy-resolved electrochemical impedance spectroscopy}

As a complementary measurement of the effect of $\beta$-phase on the hole DOS, we use a sensitive spectroscopic method for DOS mapping in organic semiconductors, namely, energy-resolved electrochemical impedance spectroscopy (ER-EIS) [81,82]. This method has previously been applied to the determination of HOMO and LUMO positions, electronic bandgap, and characteristic energy of the HOMO band tail, as well as to the characterization of defect state evolution in the bandgap of poly(3-hexylthiophene) (P3HT) induced upon degradation in air [83] or by varying layer thickness [84], and defect states in poly (silylene) materials induced by ultraviolet-light degradation [85]. Recently, the ER-EIS method, along with structural information, was used to characterize the energetic position and density of midgap trap states of the PbS nanocrystal thin film [86].

Representative ER-EIS spectra of the HOMO branch of thin glassy PFO films with and without $\beta$-phase are shown in Fig. 6. The energy scale was recalculated from the applied bias voltage to electronvolt using the $-4.66-\mathrm{eV}$ energy of the $\mathrm{Ag} / \mathrm{AgCl}$ reference electrode vs vacuum level. Note that this calibration yields HOMO energies that are deeper than those for the same polymer thin films measured using photoelectron spectroscopy and cyclic voltammetry (about 5.6-5.8 eV) $[14,87,88]$. The $g(E)$ scales inversely proportional to the measured chargetransfer resistance $R_{\mathrm{CT}}$ (see Appendix C5) and is calibrated to the DOS scale by assuming the maximum of the HOMO branch to be on the order of about $10^{21} \mathrm{~cm}^{-3} \mathrm{eV}^{-1}[89,90]$. In the spectra, we recognize, for both samples, a peak in the DOS at $-6.4 \mathrm{eV}$, with an additional feature (peak or slight shoulder) localized approximately $0.3 \mathrm{eV}$ above the DOS maximum at ca. $-6.1 \mathrm{eV}$. This band of states is more pronounced for the PFO film with intentionally introduced $\beta$-phase, which indicates its origin could be related to the presence of $\beta$-phase. If so, then the glassy sample in this 


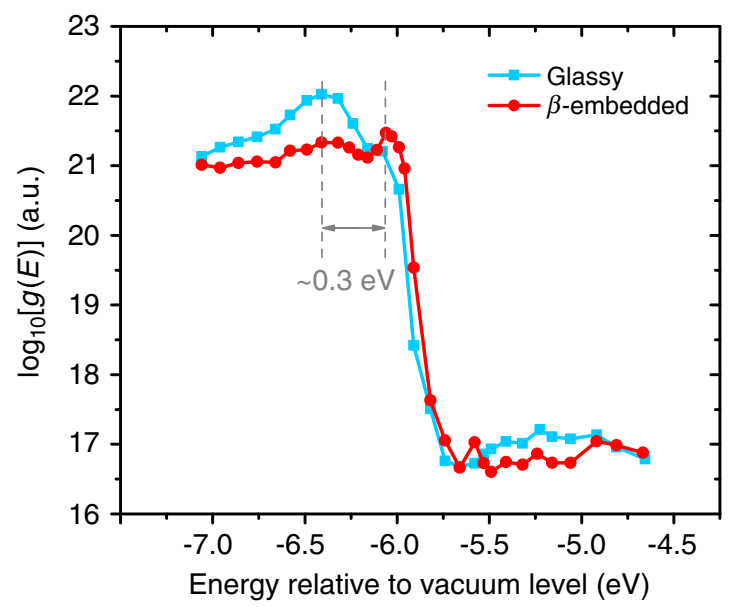

FIG. 6. Density-of-states curves of the HOMO branch measured by ER-EIS for thin films $(\sim 40 \mathrm{~nm})$ of glassy (blue) and $\beta$-phase embedded (red) PFO. The $\beta$-phase embedded sample shows a distinct satellite peak around $0.3 \mathrm{eV}$ above the HOMO peak of the glassy sample. The faint shoulder visible in the glassy sample around the position of the $\beta$-phase feature is likely due to contamination with a minute amount of $\beta$-phase developed during preparation of the glassy sample.

experiment must also contain a certain trace amount of $\beta$-phase, which is likely due to a combination of the low film thickness $(\sim 40 \mathrm{~nm})$ used for ER-EIS measurements, the effect of the rough ITO surface in seeding $\beta$-phase development, and the high sensitivity of the ER-EIS technique. The floor-level DOS deep in the bandgap is estimated as $10^{16}-10^{17} \mathrm{~cm}^{-3} \mathrm{eV}^{-1}$. This value is in good agreement with values determined from the TSC technique on a polyfluorene-type material by Renaud et al. [29].

\section{SIMULATING THE EFFECT OF $\beta$-PHASE ON THE DENSITY OF STATES AND HOLE TRANSPORT}

We have seen that introducing $\beta$-phase into glassy PFO layers results in (i) a reduction in $\mu_{h}$, to a varied extent for different measurements, and (ii) a prominent feature in the sub-bandgap space of the hole DOS as probed using transient photocurrent and electrochemical impedance spectroscopies. In order to relate the observations directly to the presence of the $\beta$-phase conformer, here we take a theoretical approach, via first modeling the effect of $\beta$-phase on the hole DOS and then simulating the electrical response of a device in different conditions.

\section{A. The effect of $\beta$-phase on the hole DOS from 1D tight-binding simulation}

In many $\pi$-conjugated polymers, the extension and stabilization of the electronic states are strongly coupled to the torsion angle $\theta$ between planes of consecutive monomers. In PFO, as with all other polymers featuring a phenyl-phenyl linkage between monomers, the ground-state backbone conformation is nonplanar, with a dihedral angle $\theta$ of around $45^{\circ}$ or $135^{\circ}$ between the adjacent monomer planes; therefore, the electronic coupling is not maximized. In the $\beta$-phase conformer, a number of consecutive monomers lie in a coplanar configuration $\left(\theta=180^{\circ}\right)$. Although such low-entropy geometry is metastable in the solid state with its total energy lying above the ground state, this conformation stabilizes the energies of charge carriers along the polymer chain.

We model the effect of such conformational features on the hole DOS as follows. First, we estimate the electronic coupling $J_{0}$ between the HOMO orbitals of consecutive, maximally coupled monomers using the counterpoise method $[42,91]$ to be $J_{0}=0.45 \mathrm{eV}$. Then, having confirmed that the coupling varies with torsion angle like $J=J_{0}|\cos \theta|$ for polyfluorenes [58], we set up a tightbinding Hamiltonian representing a one-dimensional (1D) long chain with nonzero off-diagonal elements representing the hole coupling $J$ between each pair of neighboring monomers. For chains (or segments) in the ground-state conformation, these have the value $J=J_{0}\left|\cos \theta_{0}\right|$, where $\theta_{0}$ is the equilibrium intermonomer torsion of $45^{\circ}$ (or $135^{\circ}$ ). The diagonal elements represent the monomer site energies as seen by a hole and are identical at a value that we denote as $E_{0}$ (calculated to be $-5.85 \mathrm{eV}$ relative to vacuum using density functional theory on a monomer, using the B3LYP/6-31G* functional-basis set combination) for a chain of identical monomers. To represent a degree of energetic and conformational disorder, the site energies and angles $\theta$ can be distributed around the equilibrium values. The $\beta$-phase segments are modeled as sequences with uniform site energy, also at $E_{0}$, and a maximum $J=J_{0}$ corresponding to $\theta=180^{\circ}$.

Figure S1 of the Supplemental Material [72] shows that a perfectly ordered chain with constant intermonomer torsion $\left(\theta_{0}\right.$ of $\left.45^{\circ}\right)$ leads to a DOS with the characteristic form for a one-dimensional band (sharp maxima in the DOS at the extremes of the distribution). Introducing energetic or conformational disorder (or both) broadens the DOS to introduce tail states below the onset of the DOS (see Fig. 7). The effect of introducing an ordered $\beta$-phase segment of length $N$ (we use $N \geq 20$, guided by experimental evidence in Ref. [92]) is, in contrast, to introduce distinct states lying well below the onset of the one-dimensional band (see the SM [72], Sec. I, Fig. S1, for the effect of a $\beta$-phase segment of length 20 on the DOS of an ordered 100-mer). Figure 7 depicts the same phenomena calculated for a system that contains disorder of width $\sigma_{E}=0.05 \mathrm{eV}$ in the site HOMO energies and disorder of $\sigma_{\theta}=0.1$ radians in the torsion angles of the glassy parts. In the disordered system, the feature in the density of states due to the $\beta$-phase is still prominent and is separated by around $0.25 \mathrm{eV}$ from the HOMO of the $\beta$-phase free chain.

The energetic position of the $\beta$-phase DOS onset compares well with the position of the feature introduced 

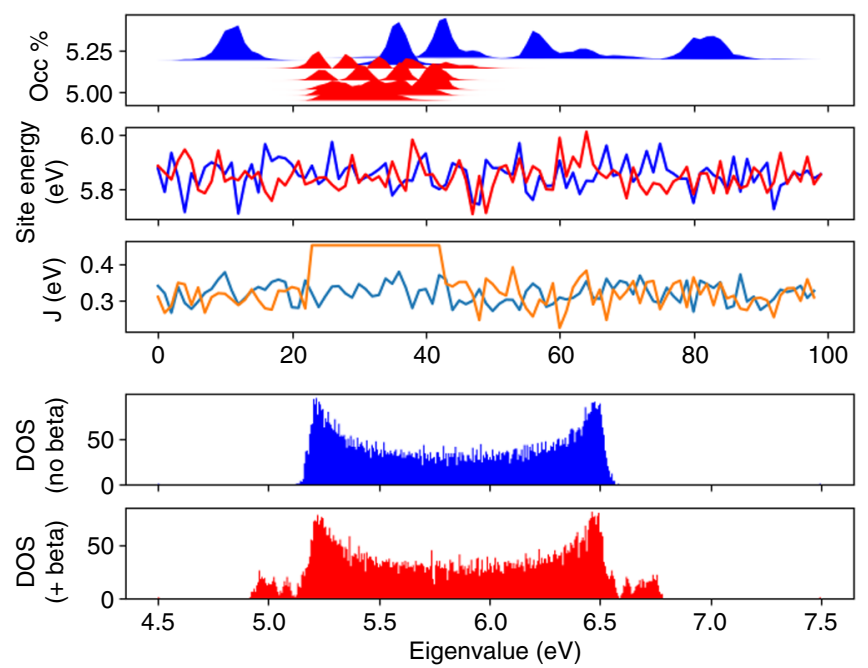

FIG. 7. Top panel: Probability density (i.e., $|\psi|^{2}$ ) for the lowest five eigenfunctions $\psi$ for an energetically and torsionally disordered $\beta$-phase free 100-mer chain (blue), and a disordered chain containing a 20 -mer of $\beta$-phase (red), with the energetic disorder width $0.05 \mathrm{eV}$ and the torsional disorder width 0.1 radians. Note that the lowest eigenfunctions for the system containing the $\beta$-phase segment are localized in the planarized segment. Second panel: Site HOMO energies for the disordered $\beta$-phase free (blue) and $\beta$-phase embedded (red) chains. Third panel: Intersite (intermonomer) electronic couplings for the disordered $\beta$-phase free (light blue) and $\beta$-phase embedded (orange) chains. The $\mathrm{x}$ axes of the first three panels present the spatial extent along the 100-mers. Fourth panel: Density of states obtained by summing over all eigenvalues for the disordered $\beta$-phase free chain (blue). Bottom panel: Density of states obtained by summing over all eigenvalues for the disordered $\beta$-phase embedded chain (red). The $\mathrm{x}$ axes of the bottom two panels present energy in units of electronvolts.

into the experimental DOS by the $\beta$-phase and confirms that the $\beta$-phase segments will act as intrachain trap states for holes. The stabilization of the hole energy in the $\beta$-phase segment is a direct result of the increased electronic coupling within the planarized segment and of the segment length. Density functional theory calculations of the hole energy confirm that planarization could stabilize the HOMO of a fluorene octamer by around $0.20 \mathrm{eV}$ relative to that of the glassy conformer (see the SM [72], Sec. I, Table S1). This is a lower limit as longer segments will be more strongly stabilized, and it is consistent with results from the tight-binding model.

\section{B. Numerical DOS and the reproduction of transport phenomena}

We now investigate the effect of such changes in the DOS on time-resolved and steady-state transport using a continuum device model. When we consider the comparison of HDR photocurrent transients and extracted DOS maps in Fig. 5, we hypothesize that the transient knee of the $\beta$-phase embedded device at around $2 \mu \mathrm{s}$, which determines the value of the hole transit time used to calculate TOF mobility, may be significantly influenced by the release of charge carriers from and the recapture of carriers into trap levels introduced by the presence of $\beta$-phase. This process is likely to persist until the drop in photocurrent occurs upon the charge packet reaching the counter electrode, which is compatible with the usual interpretation of TOF transients. The effects of charge transit and detrapping thus need to be resolved. It is, however, difficult to convert TOF transients around the knee into a DOS profile directly using Eqs. (1) and (2) because the knee lies close to the pretransit region where Eqs. (1) and (2) are not valid. Furthermore, Eqs. (1) and (2) often predict broader features in the DOS than really exist, due to the assumption that there is no thermal broadening effect on the demarcation energy $E^{*}$ [as in Eq. (2)]. Therefore, to relate the photocurrent transient to the underlying DOS distribution, a more complex model is needed.

To help us interpret the TOF transients and other electrical measurements, we adapt an open-source 1D numerical driftdiffusion model named General-Purpose Photovoltaic Device Model [93]. This model solves the bipolar driftdiffusion equations in the time domain to take account of charge-carrier transport across the device, and Poisson's equation to account for electrostatic effects [11]. We approximate the motion of mobile charge carriers using two parabolic bands, one to represent the effective DOS for free electrons and the other to represent the effective DOS for free holes. Position space is discretized using 50 evenly spaced, finite-difference mesh points (a value that is adequate to generate device response with high numerical accuracy; as shown in the SM [72], Sec. I, Part B, Fig. S2). To account for energy-dependent charge-carrier trapping and recombination, at each mesh point (i.e., a 0D energy "slice"), we introduce a set of 80 discretized trap levels spanning $0.8 \mathrm{eV}$ (i.e., $10 \mathrm{meV}$ per interval) beneath the LUMO and another set of such trap levels above the HOMO; at each level, the DOS for that energy interval is defined, and each trap level at an individual mesh point can be occupied by the relevant charge carriers up to the limit of the DOS for that interval. For each trap interval, we solve the Shockley-Read-Hall rate equations in the time domain. Thus, we are able to resolve the flow of carriers across the device, as well as the charge density in both position and energy spaces as a function of time without assuming quasiequilibrium to describe the occupancy of states. This and similar approaches have previously been applied to organic semiconductor devices to model experimental data, including steady-state $J-V$ measurements [94,95], small-signal ac response [96], ultrafast pump-probe measurements [97], and other nonequilibrium transient transport phenomena $[95,98]$.

To first model the hole DOS for glassy PFO, we combine an exponential tail of $E_{\mathrm{ch}}=75 \mathrm{meV}$, as suggested by the long-time limit of the DOS mapped from the HDR TOF transients, and a Gaussian centered around the transport 
energy level with a breadth of $75 \mathrm{meV}$. The peak magnitude of the Gaussian and the trap density of the exponential tail at the band edge are varied until good fits are obtained for the glassy TOF and steady-state $J-V$ results. We note that such a composite DOS has previously been proposed in the literature for other material systems [7,11-13,99-101]. The DOS of the $\beta$-phase embedded PFO was then modeled by superimposing a narrower Gaussian of $5 \times 10^{20} \mathrm{~cm}^{-3} \mathrm{eV}^{-1}$ peak height and $45 \mathrm{meV}$ breadth, centered at $270 \mathrm{meV}$ above the hole transport level. In obtaining these $\beta$-phaserelated parameters, the depth of this feature was first assigned as $0.3 \mathrm{eV}$ relative to the HOMO mobility edge, according to the HDR TOF inferred DOS [Fig. 5(b)] and the ER-EIS results (Fig. 6); its height was set at about onetenth of the main Gaussian peak-a fraction inferred from the ratio of the glassy to $\beta$-phase $\mathrm{S}_{0} \rightarrow \mathrm{S}_{1}$ 0-0 transition peaks in the UV-Vis absorption spectrum [see Appendix D, Fig. 18(a); we also factor in the minor difference in the reported oscillator strength $f_{\text {osc }}$ of glassy and $\beta$-phase conformations, which is of about $1: 1.08$ [102] at their respective absorption maxima]; the initial assignment of the breadth was $36 \mathrm{meV}$ as estimated from the inhomogeneous broadening of the $\beta$-phase $\mathrm{S}_{0} \rightarrow \mathrm{S}_{1}$ 0-0 absorption profile [primarily due to the varying $\beta$-phase segment lengths; see Appendix D, Fig. 18(a)]. Guided by our experimental findings, such initial parametrization of the $\beta$-phase DOS feature already predicts well its impact on hole transport in a qualitative manner, that is, inducing a strongly delayed knee ( $t_{\text {tr }}$ increased by about 200-fold) for the hole photocurrent transient under a controlled bias of $10 \mathrm{~V}$ while reducing the forward-bias hole-only current density level by a factor of less than 5 . To better match the experimental and simulated TOF transients and steady-state $J-V$ response for the $\beta$-phase embedded series, the three parameters for the narrow Gaussian were further finetuned, all by relatively small amounts. The resulting numerical DOS functions proposed for glassy and $\beta$-phase embedded PFO are shown in Fig. 8. Further device modeling details, including other numerical constants chosen for the model, are provided in the Supplemental Material [72], Sec. I, Part B.

Figure 9 compares experimental HDR TOF transients with transients simulated using the energy-resolved driftdiffusion model with the aforementioned numerical DOS functions, under the same conditions as the experiment (including, e.g., temperature, applied bias, and film thickness). The model closely reproduces the positions of features in the glassy PFO experimental TOF curves and the long-time slope. Upon addition of the narrow Gaussian representing $\beta$-phase to the model, the simulated TOF transient develops a peak at around $2 \mu \mathrm{s}$, exactly as is observed experimentally. We also note from Fig. 9 that the simulated photocurrent transient curves cross beyond about $10 \mu \mathrm{s}$, in contrast to the experimental data. We postulate that this different behavior may result from different levels

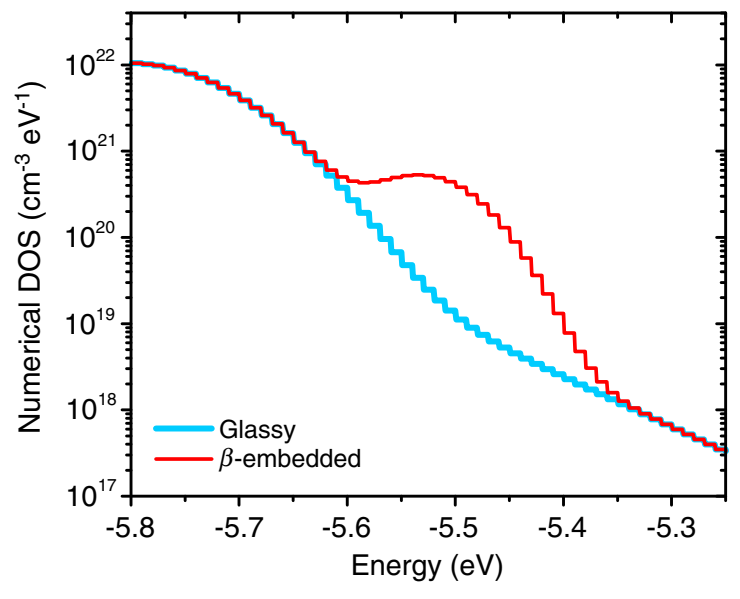

FIG. 8. Numerical hole DOS functions proposed in the driftdiffusion modeling, for glassy PFO (blue curve) and PFO containing $\beta$-phase (red curve), respectively. This set of model DOS is a simplified picture of realistic distributions; however, it nonetheless captures the key features. As an approximation due to relatively low content of $\beta$-phase, a direct superposition for constructing the $\beta$-embedded DOS is applied. The numerical $\beta$-phase feature is centered at $270 \mathrm{meV}$ above the transport level, featuring a breadth of $45 \mathrm{meV}$ and a $5 \times 10^{20} \mathrm{~cm}^{-3} \mathrm{eV}^{-1}$ peak height. The graphs are plotted as piecewise-constant functions to represent the discretization in energy space of the DOS distributions that are used in the numerical model.

of carrier occupancy in the experimental case due to unintentional variations in the laser pulse intensity, whereas in the simulation case, laser pulse photon flux is kept the same. The crossing observed in the simulated case is

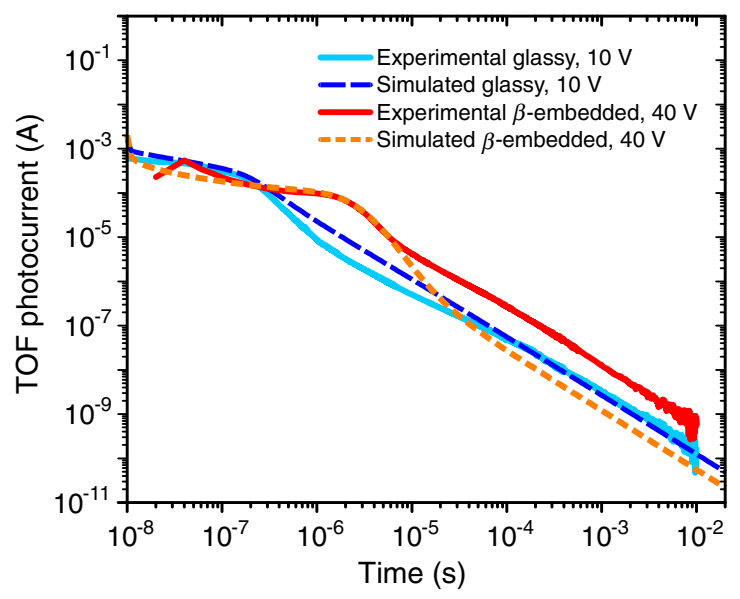

FIG. 9. Simulated (dashed lines) and experimental (solid lines) HDR TOF transients for glassy (blue) and $\beta$-phase embedded (red) HMW PFO devices at RT. The experimental traces are reproduced from Fig. 5(a). The model reproduces positions of transient features and trends for both device types. The $\beta$-phase is introduced by adding a Gaussian sub-bandgap state as depicted in Fig. 8 and with no other parameter changed relative to the glassy case. Note that the transients are taken at different applied biases for the two systems. 


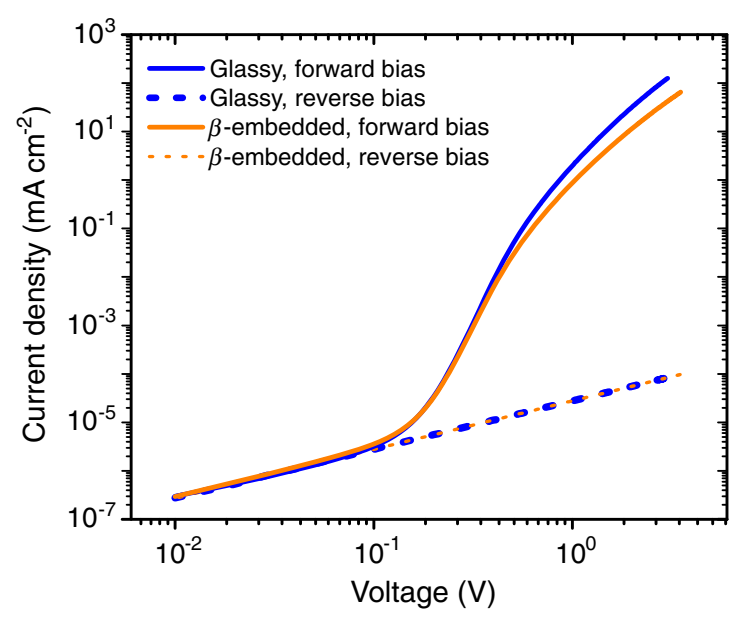

FIG. 10. Simulated steady-state dark current density-voltage curves for glassy (blue) and $\beta$-phase embedded (orange) PFO hole-only devices. The model reproduces the slight reduction in forward-bias current density that is observed experimentally, indicative of a lower steady-state $\mu_{h}$ for $\beta$-phase embedded than for purely glassy hole-only devices.

expected because the additional band of states in the DOS of the $\beta$-embedded device resulting from the $\beta$-phase defect means that fewer carriers are generated in the deep tail than in the glassy device, resulting in a lower level of transient photocurrent in the long-time limit. We stress that the only differences between the two simulations in Fig. 9 are (i) the addition of the narrow Gaussian deep trap feature and (ii) change of the bias voltage according to the respective experimental settings. No other simulation parameters were altered. The appearance of this strongly delayed knee in the modeled curve as seen in the experimental one suggests that the experimental feature is very likely caused by charge-carrier transport events involving the $\beta$-phase trap

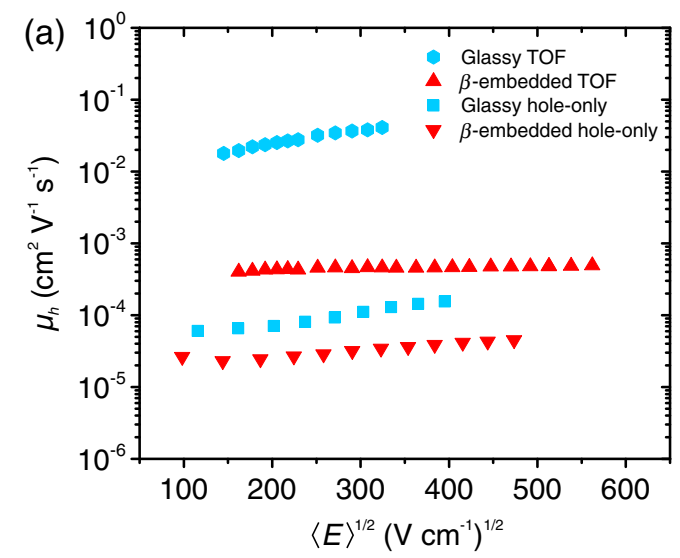

levels. The model includes charge-carrier trapping, detrapping, and transport; thus, it is likely to be due to a combination of these effects. Extraction of a transit time from the simulated curves while assuming that this knee is due to charge-carrier collection leads to an estimate of mobility for the $\beta$-phase embedded device that is around 2 orders of magnitude lower than for the glassy device, as found experimentally and demonstrated below.

Figure 10 shows the simulated steady-state dark $J-V$ curves for comparison with the experimental data in Fig. 3, obtained using exactly the same model for the material parameters as those used to model the TOF results, and via allowing for the different contact barriers (one Ohmic contact for dark $J-V$ rather than two blocking contacts for TOF) and different PFO layer thickness of the hole-only devices relative to the TOF devices ( $135 \mathrm{~nm}$ for dark $J-V$ rather than $1.8 \mu \mathrm{m}$ for TOF). The $J-V$ curves are obtained from the steady-state dark current response of the modeled device to a sweep of forward-bias voltage. Remarkably, the steady-state model predicts that $\beta$-phase has only a mild effect on the $J-V$ response relative to the glassy case, exactly as seen experimentally in Fig. 3, even though the same model of the two materials reproduces a dramatic effect on the TOF photocurrent transients.

Figure 11(a) presents a Poole-Frenkel form summary of the $\mu_{h}$ values extracted from TOF devices and impedance spectroscopic measurements on hole-only devices. Figure 11(b) plots the corresponding simulated results where the $\mu_{h}$ of hole-only devices are extracted from the simulated steady-state $J-V$ response. The simulated TOF $\mu_{h}$ values are extracted by analyzing the modeled transients identical to the approach applied for the experimental transients, while the simulated dc $\mu_{h}$ values for hole-only devices are derived by multiplying the band $\mu_{h}$ assigned to the model by the ratio of free to total (i.e., free

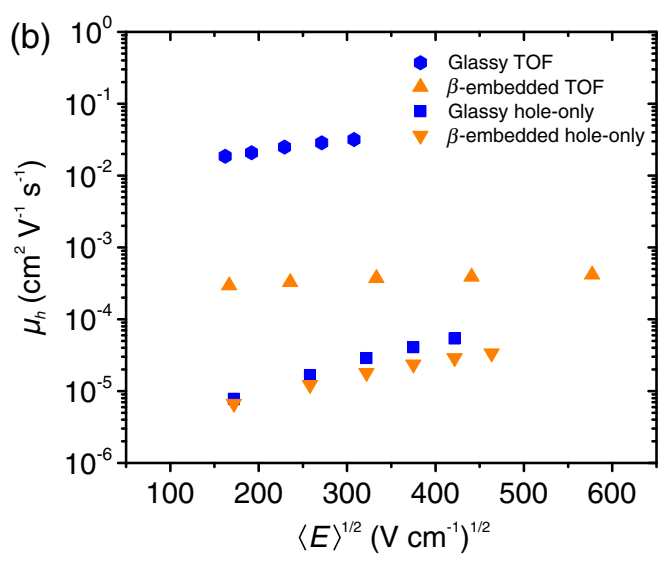

FIG. 11. (a) Summary of experimental values of HMW PFO $\mu_{h}$ at RT, as presented in Poole-Frenkel form. The $\mu_{h}$ values are reproduced from Fig. 2(b) for TOF and from Fig. 4 for impedance spectroscopy measurements on hole-only devices. (b) Summary of PFO $\mu_{h}$ values extracted from simulated response of TOF and hole-only dark $J-V$. The simulated TOF $\mu_{h}$ values are extracted by analyzing the simulated transients in the same way as for the experimental transients, while the simulated dc $\mu_{h}$ values for hole-only devices are derived by multiplying the band $\mu_{h}$ by the ratio of free charge density to total (free and trapped) charge density at a mesh point right next to the counter electrode along the $1 \mathrm{D}$ device space. 
plus trapped) charge density (as proposed by Refs. [2,103]), at a mesh point right next to the hole-extracting electrode along the 1D device space (see the SM [72], Sec. I, Fig. S3 for simulated spatially resolved profiles of densities of free and trapped charge carriers within the hole-only devices). The model reproduces the experimental results well in terms of both magnitude of effects and trends. Notably, the simulated TOF transients for the $\beta$-phase embedded device lead to an apparent $\mu_{h}$ up to 2 orders of magnitude lower than that of the glassy case, while the simulated dark steady-state response leads to a mobility reduction of only a factor of about $2-3$. We further note that the value of the TOF $\mu_{h}$ extracted from the numerical simulation is not sensitive to the active layer thickness, as shown in Fig. S4 of the SM [72]. The discrepancy in the $\mu_{h}$-field-dependence slopes from hole-only devices between Figs. 11(a) and 11(b) (with simulated ones showing a stronger field dependence than the experimental counterparts) may primarily be due to the fact that the diffusion contribution of holes was not excluded in extracting the experimental $\mu_{h}$ (a, strictly speaking, driftonly term), hence giving rise to values higher than realistic $\mu_{h}$ especially in the low-field range where carrier diffusion prevails over drift. The observation may also be caused by a minor discrepancy in the estimated $V_{\mathrm{bi}}$ from $C-V$ characteristics of the hole-only devices (see Appendix G, Part 2) relative to the actual value, thus leading to a slight overcorrection for the field dependence.

\section{RECONCILING THE CONTRADICTORY EFFECTS OF $\beta$-PHASE ON HOLE TRANSPORT}

\section{A. Significance of accounting for carrier relaxation in interpreting transport phenomena}

We have established that a consistent picture of the density of states due to the $\beta$-phase conformational defect
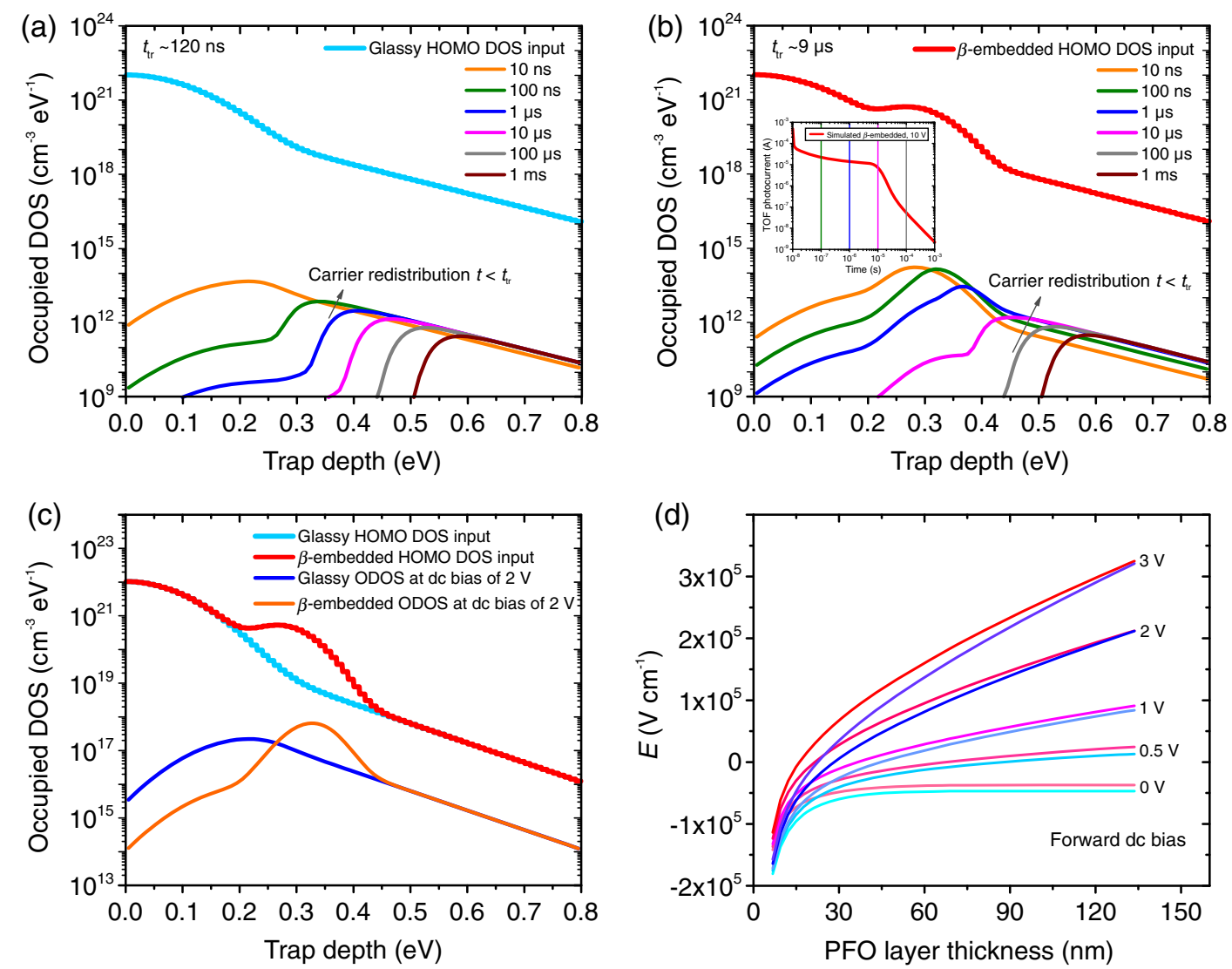

FIG. 12. Plots of the simulated occupied density of states (ODOS) at a point in close proximity (at the 5 th out of a total 50 mesh points) to the front anode of a TOF device at logarithmically spaced times after laser excitation for (a) a glassy PFO device with $V_{\text {bias }}=10$ and $t_{\text {tr }} \sim 120 \mathrm{~ns}$ and (b) a $\beta$-phase embedded PFO device with $V_{\text {bias }}=10$ and $t_{\text {tr }} \sim 9 \mu$ s. The inset shows the modeled hole photocurrent transient (red curve). Colored vertical lines in the inset mark the times following laser excitation, in accordance with the figure legend. The arrows in subplots (a) and (b) denote carrier redistribution into deep states that occurs in the pretransit regime (when $t<t_{\text {tr }}$ ). (c) ODOS of glassy and $\beta$-phase embedded PFO hole-only devices modeled at a forward dc bias of $2 \mathrm{~V}$ at the midpoint along the active layer thickness. The thick, piecewise-constant functions in subplots (a), (b), and (c) represent numerical hole DOS for glassy (sky blue) and $\beta$-phase embedded (red) PFO. (d) Simulated electrostatic field profiles across the glassy (blue-color sets) and $\beta$-embedded (red-colour sets) PFO active layers in hole-only devices under a range of forward dc biases. 
can account for the different effects on PFO device response from different (transient, impedance, and steady-state) transport probes. It remains to establish how electronic transport (and apparent mobility) is related to carrier dynamics in the different measurements. To do this, we first simulate TOF transients with and without $\beta$-phase at a controlled external bias of $10 \mathrm{~V}$ and temperature of $295 \mathrm{~K}$. We plot the carrier distribution within the sub-bandgap states at particular time points, by considering a slice at the 5 th out of a total 50 mesh points of the device (counting from the anode toward the cathode). Figure 12(a) plots the hole density in the DOS for such a slice of a glassy device at times spaced logarithmically from $10 \mathrm{~ns}$ to $1 \mathrm{~ms}$ after photoexcitation. Beyond about $1 \mu$ s (i.e., after $t_{\mathrm{tr}} \sim 120 \mathrm{~ns}$ ), the carrier distribution shows a relatively sharp edge distinguishing occupied from unoccupied states and minimal carrier redistribution into deep traps due to retrapping, as theory predicts for a post-transit photocurrent transient under a strong field $[2,4]$. In contrast, when the Gaussian band of localized states associated with the $\beta$-phase is present in the DOS [Fig. 12(b)], there is no sharp edge to the carrier distribution until more than $10 \mu$ s (i.e., after $t_{\mathrm{tr}} \sim 9 \mu \mathrm{s}$ ), and at earlier times, a significant fraction of the states lying between 0.2 and $0.35 \mathrm{eV}$ above the band edge is populated. It is the charge-carrier interaction with these trap states-through trapping and release-that controls the onset of the kneelike feature $\left(t_{\mathrm{tr}} \sim 9 \mu \mathrm{s}\right)$. The pretransit plateau corresponds to a window of time during which repetitive charge retrapping and detrapping occur from the $\beta$-phase defect such that the population of mobile holes is mostly maintained (apart from a small carrier redistribution into deep tail states), as illustrated in the inset to Fig. 12(b). This leads to the much-delayed arrival of the fastest charge carriers at the counter electrode relative to the $\beta$-phase-free (glassy) case.

This analysis allows us to resolve the difference between the mobilities measured using transient and steady-state methods for the same material system. The DOS for the glassy and $\beta$-phase embedded devices are largely similar and differ only in the presence-for the $\beta$-phase embedded case - of a small density of trap states concentrated at a level around $0.3 \mathrm{eV}$ above the hole transport edge. The contribution to current at each point in the device is controlled by the density of free holes at that point. The density of free holes is controlled in turn by the activation of the most energetic (relatively "hot") charge carriers in the sub-bandgap DOS to the transport level with the result that current is higher when the activation barrier is lower (DOS filled to a higher level) or when the carrier population at the highest filled level is higher. To understand the differences in the effect of $\beta$-phase on the measured mobility, we need to understand the difference in the transient and steady-state measurements.

In steady-state conditions, such as in dark $J-V$ measurements, the filling of the density of states varies across the device while a uniform current density is maintained by variations in the local electrostatic potential. The effect of $\beta$-phase is to increase the DOS in a certain energy window above the transport level such that for any charge-carrier density, sufficient to fill the DOS up to or beyond the $\beta$-phase defect level, more of the charge carriers are located further from the hole transport level; the density of free holes is lower, and therefore the current is lower. The presence of the $\beta$-phase defect has a noticeable effect on DOS filling within the hole-only devices under forward bias, as illustrated by plots of the occupied DOS in Fig. 12(c), indicating that the profile of trapped carriers in the $\beta$-phase embedded hole-only device is overall "cooler" than that of the glassy device. This effect suppresses the density of free holes in the bulk of the device by a factor of between about 2 and 10 (depending on position; see the SM [72], Sec. I, Fig. S3) relative to the glassy case, and it affects the electric-field distribution as shown in Fig. 12(d). The overall effect is thus to reduce the hole mobility.

The transient photocurrent measurement made by TOF differs in a few respects. First, because of the low optical excitation density and the absence of electrically injected charges, the level of DOS filling is not only much lower but it also varies more strongly across the optically thick layer (especially soon after the photogeneration of carriers). Second, the excess charge carriers are not fully relaxed in the DOS during the timescale of the measurement-their energetic distribution starts overall hot and progressively cools down, leading to an effective carrier mobility that decays with time. Third, mobility is therefore estimated from the time-dependent photocurrent, which is effectively a reflection of the change in the occupied DOS with time, rather than from an average photocurrent that reflects different levels of occupancy. Because the time-dependent photocurrent is sensitive to features in the DOS, and the DOS filling level does not change strongly across the bulk of the device once it has entered the post-transit regime, a strong change in photocurrent corresponds to a distinct subbandgap DOS peak (like the $\beta$-phase) as the DOS empties by carrier detrapping. In addition, when such a distinct feature is present (and provided it is relatively shallow), the carriers undergo a much larger amount of repetitive trappingdetrapping events during the prolonged pretransit time, thus leading to more redistribution of carriers into deeper traps [compare carrier redistribution in Figs. 12(a) and 12(b)]. The analysis of TOF transients is based on the idea that the induced photocurrent drops when charge carriers reach the opposite electrode; thus, a qualitative change in photocurrent is associated with a transit time. However, the presence of peaks in the DOS can lead to significant changes in the photocurrent due to capture and release of trapped carriers. These effects produce changes in the shape of the photocurrent such as a clear and strongly delayed kneelike feature; hence, the apparent experimental observation of "nondispersive" [104], or even seemingly "trap-free" [105], 
transport. It is noteworthy that due to the reciprocity between time and frequency, a profound influence of $\beta$-phase on carrier dynamics is also visible in the frequency-resolved capacitance-voltage response of the hole-only devices (see Fig. 24 in Appendix G). In addition, thanks to a higher volume content of $\beta$-phase induced, the LMW devices amplify the effect of $\beta$-phase by featuring strong "cusps" in their TOF transients, as shown in Fig. S8(a) of the Supplemental Material [72]. This observation supports the idea that the photocurrent feature reflects charge-carrier trapping and release events associated with the distinct conformational defect. In the case of the $\beta$-phase feature in $\mathrm{PFO}$, the effect on the photocurrent transient of the chargecarrier interaction with the $\beta$-phase defect is to shift the transit time to longer times, thus leading to a substantially lower value of the estimated mobility. This result demonstrates that all photocurrent transients, in general, reflect a combination of charge-carrier trapping, detrapping, recombination, and transport processes, and therefore, it is difficult to rely on simple analytical models to interpret the device response, especially in the presence of nontrivial density-ofstate distributions. We further note that the assumed relationship between photocurrent evolution and mobility that is normally used for TOF is not always appropriate when there are strong peaks in the sub-bandgap DOS. (For more details, see Sec. IV of the SM [72], where we generalize the interplay between such sub-bandgap DOS and band mobility on the photocurrent shape.) If a true estimate of the effect of $\beta$-phase on steady-state hole transport in a device were needed, the analysis used here for the steady-state measurements should yield a more realistic estimate, while the large effect inferred from the TOF method is overestimated. We also reiterate that the TOF transient at any given time depends on the instantaneous carrier distribution profile within the DOS, which is a function of chargecarrier dynamics, the carriers' history, and the given DOS. Therefore, a detailed numerical model is required to understand the precise shape of the transient in both earlyand late-time limits, especially when the DOS shape is complex.

Next, we comment on the difference in magnitude between the hole mobilities inferred from glassy PFO devices using the TOF method (TOF $\mu_{h}=2-4 \times 10^{-2} \mathrm{~cm}^{2} \mathrm{~V}^{-1} \mathrm{~s}^{-1}$ ) and using the impedance method (steady-state $\mu_{h}=$ $0.6-1 \times 10^{-4} \mathrm{~cm}^{2} \mathrm{~V}^{-1} \mathrm{~s}^{-1}$ ) since neither measurement is affected by the $\beta$-phase defect. Higher mobility values have previously been reported using TOF when compared to vertical-mode steady-state techniques, in disordered molecular materials [106-108]. The reason is likely to be that in a disordered system, the fastest carriers that determine the appearance of a transit time in TOF (we recall their timedependent carrier mobility through progressive thermalization) are normally much faster than the mean velocity that would characterize the carrier velocity distribution for the same device in the steady state.
This discussion reveals clearly the critical effect of the choice of measurement technique on the meaning of an extracted mobility value. Figure S5 of the Supplemental Material [72] summarizes how the reported $\mu_{h}$ in PFO varies by orders of magnitude depending on the measurement technique as well as the preparation of the sample. The variations in the values of $\mu_{h}$ reported previously for PFO are likely to result partly from the unintentional presence of different phases (such as the $\beta$-phase) in the devices, as well as the different assumptions underlying analyses for different techniques. This observation underlines the fact that the value of a mobility measurement is only meaningful in the context of the assumptions underlying the measurement technique used. For example, a steady-state measurement is relevant for mobility in a device that is operated in the steady state under similar injection levels, while a value of mobility measured under conditions far from the intended operating conditions of interest may not be meaningful.

\section{B. Multiple-trapping versus polaronic conduction: Insights from previous studies of hole transport in PFO}

Finally, we consider our results in the context of prior reports of the hole transport in PFO. Before doing that, we briefly consider the different models of microscopic charge transport. We have used a multiple-trapping model where charge-carrier activation is controlled by the energy difference between a charge carrier in a trap state and the transport level. In the case of organic semiconductors, it is also common to use a polaronic transport model where charges must overcome a polaron binding energy, as well as any difference in site energy, in order to move. This additional activation energy due to polaron binding, in the weak-coupling limit [109], is given by $\lambda / 4$, where $\lambda$ is the reorganization energy. In this limit, $\lambda$ is large compared to the electronic coupling between transport sites and comparable with or larger than the disorder in transportsite energy. The presence of a significant polaron binding energy would affect the temperature dependence of mobility but would not affect the relationship between the shape of the DOS and the photocurrent transient at a given temperature. Our results may be consistent with polaronic transport, but since we work within a narrow temperature range of $16 \mathrm{~K}$, we cannot conclude from our data whether polaronic transport is relevant or not.

Several previous works placed the TOF $\mu_{h}$ of roomtemperature "as-cast" films of PFO at around, or marginally over, $2 \times 10^{-4} \mathrm{~cm}^{2} \mathrm{~V}^{-1} \mathrm{~s}^{-1}[69,104,110]$. Judging from the device preparation conditions used, it is very likely that those films contained $\beta$-phase [79]. In support of this idea, the previously reported TOF $\mu_{h}$ hole mobilities agree well with the value presented here for $\beta$-phase embedded PFO. Kreouzis and co-workers [69] assigned the transport in RT as-cast (nonannealed) PFO layers to a polaronic hopping mechanism [111] on account of the thermally activated 
nature of the zero-field hole mobility $\left[\ln \left(\mu_{h 0}\right) \propto T^{-1}\right]$, wherein the activation energy $E_{a}$ would be enhanced by the reorganization energy. In contrast, uncorrelated hopping within a Gaussian DOS would have yielded a TOF $\mu_{h}$ varying like $\ln \left(\mu_{h 0}\right) \propto T^{-2}$ [6] rather than $\ln \left(\mu_{h 0}\right) \propto$ $T^{-1}$. We can now tentatively explain this observation in terms of the effect of $\beta$-phase. The $E_{a}$ obtained from the analysis of the previously reported TOF of as-cast PFO is around $0.3 \mathrm{eV}$ [69]. This value closely matches the estimated depth $(\sim 0.3 \mathrm{eV})$ of the sub-bandgap $\beta$-phase defect in this study, and the $E_{a}$ for our $\beta$-phase embedded device as shown in the Arrhenius plot in Fig. S6 of the SM [72]. It is therefore plausible that the transport behavior reported in Ref. [69] was dominated by the thermal activation of holes out of $\beta$-phase traps, rather than by a combination of Gaussian disorder and polaron activation; also, the size of the polaron binding energy, if present, may be smaller than deduced in that study. See the SM [72], Sec. II, Part B for more discussions.

\section{CONCLUSIONS}

In this study, we have attempted to understand and address the impact of conformational defects on the density of states and charge transport in $\pi$-conjugated polymers, taking the fully extended, planar-zigzag $\beta$-phase segment in amorphous PFO as an example. By applying a HDR TOF photocurrent technique, we have probed the density of trap states extending far (up to $\sim 0.55 \mathrm{eV}$ ) above the hole transport level of the polymer, and we have shown that the DOS is affected by the presence of the $\beta$-phase conformer through the addition of a set of hole states approximately $0.3 \mathrm{eV}$ above the hole transport level. The validity of the DOS obtained by HDR TOF is confirmed using a complementary technique based on energy-resolved electrochemical impedance spectroscopy. Moreover, the energetic position and sharpness of the defect states is consistent with the simulated effect of backbone planarization on the HOMO energy in oligofluorenes.

We have also exploited the PFO system with the presence or absence of $\beta$-phase to reconcile apparent disagreement in hole mobility values extracted using different experimental techniques. Specifically, we found that while the hole mobility probed by the TOF photocurrent appears to be up to 2 orders of magnitude lower for $\beta$-phase embedded than for neat glassy PFO, the steadystate value based on hole-only devices is only a factor of $2-5$ times lower. We rationalize this by using a macroscopic device model whereby charge carriers are resolved in terms of energy as well as position and time. We show that within this model, the different observations from time-resolved and steady-state probes can be reconciled. The underlying reason for the seemingly contradictory phenomena is the different levels of carrier relaxation or occupation within the localized states, as well as the different interpretation of currents in terms of mobility for the different measurements. To the best of our knowledge, this work accomplishes the first quantitative demonstration of a direct relationship between conformational disorder, sub-bandgap DOS distribution, and charge transport behavior in a molecular electronic material. This study also highlights the importance of applying energy-resolved models to properly analyze and interpret transient, out-of-equilibrium electronic phenomena in disordered semiconductors featuring a significant density of sub-bandgap localized states.

A somewhat surprising result of this study is that conformational variants that encourage charge-carrier delocalization (such as the $\beta$-phase in PFO) can inhibit rather than assist charge transport. This phenomenon is a consequence of the limited density of $\beta$-phase segments that can be introduced into high-molecular-weight PFO, which prevent charge percolation through the $\beta$-phase alone, and the twisted nature of the optimum ground-state conformation that results from the phenyl-phenyl linkage of the polymer. The impact of conformational variation on transport in polymers with more planar ground-state geometry would be less significant [19]. The $\beta$-phase content can be increased to some extent in low-molecular-weight polymers, as shown here, but to an insufficient degree for percolation, thus leading to an even stronger penalty on mobility.

This work demonstrates how electronic transport can be controlled via molecular conformation in organic semiconductors. Another general implication from this study is that charge-carrier mobility, as a figure of merit from experimental probes, must depend heavily on the measurement conditions and carrier concentration, and on how charge carriers reflect the DOS as they transit across a disordered solid. In the specific case of PFO, this knowledge could, in principle, be exploited by tuning of charge transport through judicious control of the $\beta$-phase content. Such optimization could have potential benefits in balancing hole and electron mobility in OLEDs, as well as in simultaneously optimizing luminescence quantum yield and the spectral definition of the blue emission. In a broader context, findings of this study can also be applied to areas concerning (i) electronic transport or charge transfer within other (macro)molecular systems, where conformational freedom plays a role, and (ii) the arrival of a unified picture of the impact of structural and energetic disorder on transport behavior in disordered semiconductors observed using different transport techniques.

\section{ACKNOWLEDGMENTS}

X.S. and J.N. gratefully thank the Engineering and Physical Sciences Research Council (EPSRC) for support via Grants No. EP/M025020/1, No. EP/P005543/1, and No. EP/K029843/1. This project has also received funding from the European Research Council (ERC) under the European Union's Horizon 2020 Research and Innovation Programme (Grant Agreement No. 742708). X. S. acknowledges funding from European COST Action 
MP1307 (StableNextSol) for inter-institutional research visits. X. S. would like to thank Professor Ji-Seon Kim and Dr. Nathan A. Chander for Raman spectroscopies on PFO layers; Professor Donal D. C. Bradley, Dr. Davide Moia, and Dr. Jason A. Röhr for fruitful discussions; and Shahid Hanif, David G. Bowler, and Stephen D. Cussell for technical support for instrumentation modifications. The research of V.N. was funded by VEGA Project No. 2/0081/18.

\section{APPENDIX A: MATERIALS}

HMW and LMW PFO materials, synthesized via the Suzuki coupling reaction, were sourced from Sumitomo Chemical/Cambridge Display Technology (CDT). The polymers underwent extensive postsynthesis purification to remove ionic impurities and catalyst residues, and they were used as received. HPLC-grade toluene $(\geq 99.8 \%$, VWR Chemicals) was used as solvent throughout the PFO sample and device fabrication. Conductive polymer poly(3,4-ethylenedioxythiophene):poly(styrenesulfonate) (PEDOT:PSS; work function 5.2 eV; Heraeus Clevios ${ }^{\mathrm{TM}}$ $P$ VP AI 4083) was selected as the interlayer on indiumtin oxide (ITO) for creating asymmetric PFO hole-only devices. Ultrathin $(\sim 5 \mathrm{~nm})$ layers of molybdenum oxide $\left(\mathrm{MoO}_{x}\right.$; purity $\geq 99.5 \%$; Sigma-Aldrich) were thermally evaporated atop polymer active layers to establish Ohmic contacts for hole-only devices. Pellets of aluminium (Al; purity 99.999\%; top metallization for time-of-flight devices) and gold (Au; 99.99\%; top metallization for hole-only devices) for thermal evaporation were obtained from Kurt J. Lesker® Company.

To extract molecular-weight properties of the PFO polymers used in this study, polystyrene-equivalent gel permeation chromatography (GPC) measurements were carried out with a Viscotek instrument comprising a pump and a degasser (GPCmax VE2001), a detector module (Viscotek 302 TDA), and three columns ( $2 \times$ PLGel Mix-C and $1 \times$ ViscoGEL GMHHRN 18055). PFO solutions (including 0.1 vol\% toluene as an end marker) were prepared in chloroform with $1.2 \mathrm{mg} \mathrm{mL}^{-1}$ concentration. To promote polymer dissolution, the solutions were heated at $50^{\circ} \mathrm{C}$ for 15 minutes. They were subsequently filtered [112] to remove any possible extrinsic dust or particulates. The columns were held at $35^{\circ} \mathrm{C}$, with a solution flow rate of $1 \mathrm{~mL} \mathrm{~min}{ }^{-1}$ set for the GPC measurements. Raw detection curves for the high- and low-MW PFO solutions are presented in Fig. 13, indicating monomodal distributions of both batches and the concurrent onset of the toluene end marker. The offset in GPC peaks visualizes the relative difference in the MW profiles of the two polymers, which are therefore dubbed HWM and LWM, respectively.

OMNISEC software (Viscotek) was employed to analyze the data using conventional calibration. Because of the higher chain stiffness of PFO, its absolute molecular weight

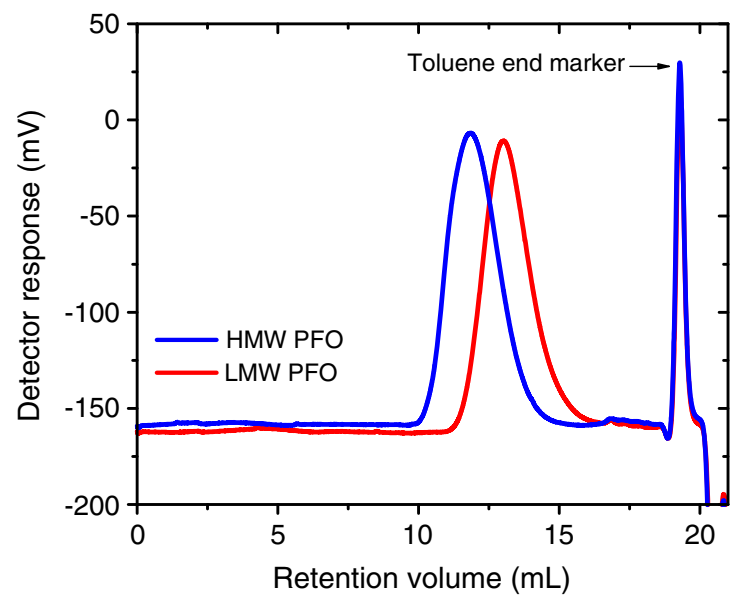

FIG. 13. Gel permeation chromatography (GPC) detection curves for both HMW (blue line) and LMW (red line) PFO solutions $\left(1.2 \mathrm{mg} \mathrm{mL}^{-1}\right.$ in chloroform with $0.1 \mathrm{vol} \%$ toluene as the end marker). Monomodal molecular distributions for both PFO batches are evident. Note that $M_{w}$ and $M_{n}$ values for both materials, as listed in Table I, were extracted from the GPC curves based on conventional polystyrene-equivalent calibration.

is a factor of 2.7 lower than the polystyrene-equivalent value [62]. The number of repeat units for the average chain length from each PFO batch was estimated from the corresponding stiffness-corrected absolute values of number-average molecular weight $\left(M_{n}\right)$ and by factoring in the molecular weight of a PFO monomer ( 388 Da). The results, along with polystyrene-equivalent weight- $\left(M_{w}\right)$ and number-average $\left(M_{n}\right)$ molecular weights, as well as the corresponding polydispersity indices ( $\left.\mathrm{PDI}=M_{w} / M_{n}\right)$, are summarized in Table I.

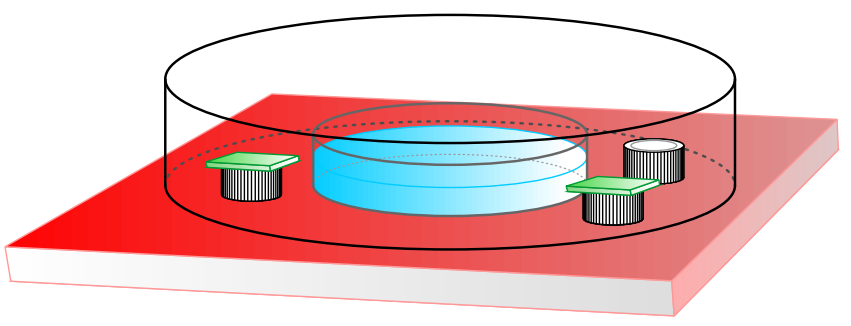

FIG. 14. False-color sketch of the solvent vapor exposure apparatus. The red stage represents the hot-plate surface kept at $50{ }^{\circ} \mathrm{C}$ during the process to promote evaporation of toluene (light blue) from a small petri dish placed atop. The lime-green pieces represent the substrates with glassy PFO layers cast uppermost. These samples are lifted above the hot-plate surface by about $1 \mathrm{~cm}$ using thermal-insulating plastic vial lids as stands. This way, we prevent direct thermal contact and thus any potential film crystallization into the $\alpha^{\prime}$-phase [113]. An enclosed chamber is achieved by placing a larger, upturned petri dish onto the hot-plate surface. The continuous vapor exposure period is set to one hour; the process is hence abbreviated as $1 \mathrm{hrSVE}$. 
TABLE I. Molecular-weight properties of the polyfluorenes.

\begin{tabular}{lccccc}
\hline \hline PFO polymer batch & $M_{w}\left(\mathrm{~kg} \mathrm{~mol}^{-1}\right)$ & $M_{n}\left(\mathrm{~kg} \mathrm{~mol}^{-1}\right)$ & PDI & $\begin{array}{c}\text { Average number of repeat units } \\
{\left[\text { from } M_{n} /\left(M_{\text {monomer }} \times 2.7\right)\right]}\end{array}$ & Supplied by \\
\hline HMW & 484.2 & 182.4 & 2.65 & 174 & Sumitomo Chemical / CDT \\
LMW & 109.5 & 42.2 & 2.6 & 40 & \\
\hline \hline
\end{tabular}

\section{APPENDIX B: SAMPLE AND DEVICE FABRICATION}

\section{Substrates}

(a) Spectrosil ${ }^{\circledR}$ B fused-silica substrates $\left(12 \times 12 \mathrm{~mm}^{2}\right.$; thickness $\sim 1 \mathrm{~mm}$; UQG Optics) were used to realize PFO thin-film samples for optical spectroscopies.

(b) For TOF and hole-only devices, as well as samples for energy-resolved electrochemical impedance spectroscopy (ER-EIS), glass/indium-tin oxide (glass/ITO) substrates $\left(12 \times 12 \mathrm{~mm}^{2}\right.$; ITO layer thickness of about 150-250 nm; ITO work function of about $4.7 \mathrm{eV}$; total thickness of about $1 \mathrm{~mm}$; PsiOTec) were used. Sheet resistance of the prepatterned ITO stripe (covering the central $8 \times 12 \mathrm{~mm}^{2}$ area) was about $10-15 \Omega / \square$ [see Fig. 15(a) for the substrate pattern]. PEDOT:PSS interlayers $(\sim 30 \mathrm{~nm})$ were treated on top (via spin coating at $3000 \mathrm{rpm}$ for $60 \mathrm{~s}$ followed by drying at $120^{\circ} \mathrm{C}$ for $15 \mathrm{~min}$ ) in the case of making hole-only devices.

Below, we provide fabrication protocols for the PFO samples and devices investigated in the present work. (a)

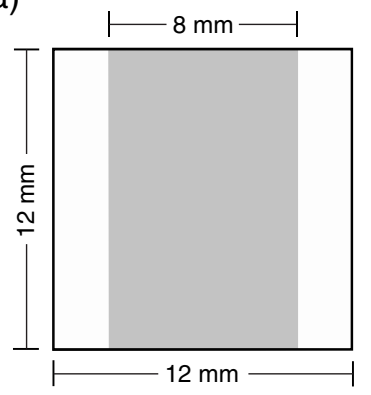

(b)

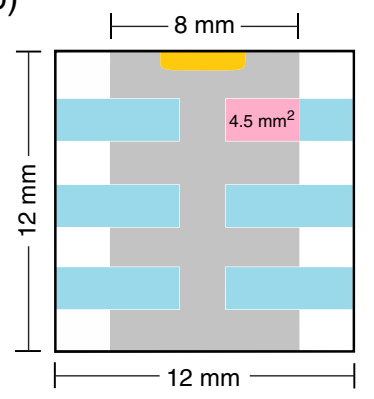

FIG. 15. Schematic top views of (a) the bare glass/ITO substrate and (b) finished devices thereon. In panel (a), the $12 \times$ $12 \mathrm{~mm}^{2}$ glass substrate is partially covered by an $8 \times 12 \mathrm{~mm}^{2}$ ITO stripe (grey). The thickness of the ITO layer varies from 150 to $250 \mathrm{~nm}$ depending on batches of manufacture. The total thickness of the substrate is about $1 \mathrm{~mm}$; in panel (b), six top electrodes (sky blue) are deposited via thermal evaporation under high vacuum $\left(\leq 10^{-6}\right.$ mbar) through a shadow mask, thereby forming six induvial devices (pink). The overlap between the top contact and ITO stripe defines the effective geometric area for each single device $\left(\sim 4.5 \mathrm{~mm}^{2}\right)$. Electrical connection to the bottom ITO electrode is established by applying conductive silver paste (orange) at either edge of the ITO stripe after physically removing the local active layer. Such a configuration allows for characterization of six devices on one substrate and hence comparison of their property-performance uniformity.

\section{Glassy PFO film deposition}

A solution of HMW PFO (7 mg ml${ }^{-1}$ in toluene) was spin coated onto thoroughly cleaned and $\mathrm{O}_{2}$-plasma-treated fused-silica substrates at $1200 \mathrm{rpm}$, yielding PFO thin films with thickness of about $65 \mathrm{~nm}$ for UV-Vis absorption measurements. To fabricate "baseline" isotropic glassy PFO films with maximally suppressed $\beta$-phase and crystalline chain segments, both the solution and substrates were placed on a hot plate set at $95^{\circ} \mathrm{C}$ (below the boiling point of toluene, $\sim 111^{\circ} \mathrm{C}$, to avoid bubbling) for at least 3 minutes immediately prior to spin coating: The total duration of steps, including (i) transfer of the preheated substrate to the vacuum chuck, (ii) pipetting hot PFO solution onto the substrate, (iii) closing the spin coater lid, and (iv) initiating the spin, was controlled to be less than $8 \mathrm{~s}$. We refer to the above process as "hot spin coating" for PFO films.

Optically thick $(\sim 1.8 \mu \mathrm{m})$ HMW glassy PFO photoconductive layers in TOF devices were prepared according to the hot spin-coating procedures described above, except from spin coating $30 \mathrm{mg} \mathrm{ml}^{-1}$ viscous HMW PFO solutions in toluene at $800 \mathrm{rpm}$ for $60 \mathrm{~s}$, followed by a 30-s "smoothing" step at $2000 \mathrm{rpm}$, onto cleaned, $\mathrm{O}_{2}$-plasmatreated glass/ITO substrates. Moreover, for these thick films, an aluminium vacuum chuck was also employed and preheated to about $95^{\circ} \mathrm{C}$, in order to promote maximally rapid solvent evaporation and thereby prevent chain relaxation during solidification [79] to form the $\beta$-phase. Likewise, LMW PFO photoconductive layers $(\sim 3-4.1 \mu \mathrm{m})$ were also cast following hot spin coating, however using 700-1200 rpm and a higher solution concentration of $\sim 70 \mathrm{mg} \mathrm{ml}^{-1}$.

For the preparation of hole-only devices, glassy PFO layers were similarly cast onto PEDOT:PSS precoated ITO/glass substrates.

Prior to the deposition of top contacts, in order to generate $\beta$-phase embedded (or " $\beta$-embedded" for short) variants, the hot-spun glassy PFO layers were further processed according to procedures described below.

\section{Inducing the $\beta$-phase by exposing glassy PFO layers to solvent vapors}

To induce planarized $\beta$-phase conformers into pristine glassy PFO layers (of both optically thin and thick), postdeposition solvent vapor exposure (SVE) was performed, as illustrated in Fig. 14. See figure caption for detailed procedures. Note that this 1-hour treatment was for 
a period significantly longer than the period of about 10 minutes estimated by Caruso and Anni in achieving the saturated formation of $\beta$-phase chain segments in 400-nm toluene-vapor-exposed films [64].

Subsequent to film deposition and "1hrSVE" postdeposition treatments, the films were desiccated over several hours to remove any residual trapped solvent. Film thickness was determined using a DektakXT ${ }^{\mathrm{TM}}$ surface profilometer.

\section{Thermal evaporation of top contacts}

For the TOF devices, about 100-nm-thick Al counter electrodes were deposited via thermal evaporation at $\leq 10^{-6}$ mbar through a shadow mask onto optically thick PFO films, yielding stacked structures with a geometric device area of about $4.5 \mathrm{~mm}^{2}$ defined by the electrode overlap regions (see Fig. 15 for details).

The hole-only devices feature likewise geometric layouts while having ultrathin $(\sim 5 \mathrm{~nm}) \mathrm{MoO}_{x}$ interlayers further capped by about 100-nm Au as top metallization.

\section{APPENDIX C: SETUPS OF (OPTO)ELECTRICAL PROBES}

\section{Time-of-flight photocurrent charge-carrier motility}

Photogeneration of the charge carriers was delivered by pulsed frequency-tripled Nd:YAG laser excitation $\left(\lambda_{\mathrm{ex}}=355 \mathrm{~nm}\right.$; pulse width $\sim 5 \mathrm{~ns}$; Quantel) through the ITO front anode. A Keithley high-voltage source-measureunit (SMU) 237 was used for applying a variable dc bias voltage across TOF devices. Typical dc dark current levels are less than $25 \mathrm{pA}$ at $50-\mathrm{V}$ bias for the PFO TOF devices, indicating excellent blocking properties of the chosen contacts. Photocurrent transients were recorded using a Tektronix ${ }^{\circledR}$ TDS 3052 digital storage oscilloscope (or scope for short) with its $0.5-\mathrm{GHz}$ full-bandwidth setting. Neutral-density filters were applied to limit the intensity of the incident laser beam to $Q / Q_{0}<10 \%$ (and less than $15 \%$ in the HDR measurement scenario), where $Q_{0}$ is the biasdependent amount of charges held across the contacts of the sandwichlike TOF devices and $Q$ the population of photogenerated charges. Figure 16 shows a schematic of the TOF photocurrent setup.

To capture fast transients featuring, e.g., sub-100-ns transit time, $50 \Omega$ channel termination was chosen (which matches the $50 \Omega$ coaxial cable characteristic impedance) and with a $50 \Omega$ source impedance fitted onto the Keithley SMU 237 for optimized electrical response. Data acquisition was externally triggered by the laser Q-switch synchronization signal. When necessary, transients were recorded with a 256- or 512-averaged sampling mode for an enhanced signal-to-noise ratio (SNR).

During the TOF measurement at room temperature (typically at about $295 \mathrm{~K}$ ), devices were housed inside a hermetic, pure nitrogen filled and electrically grounded

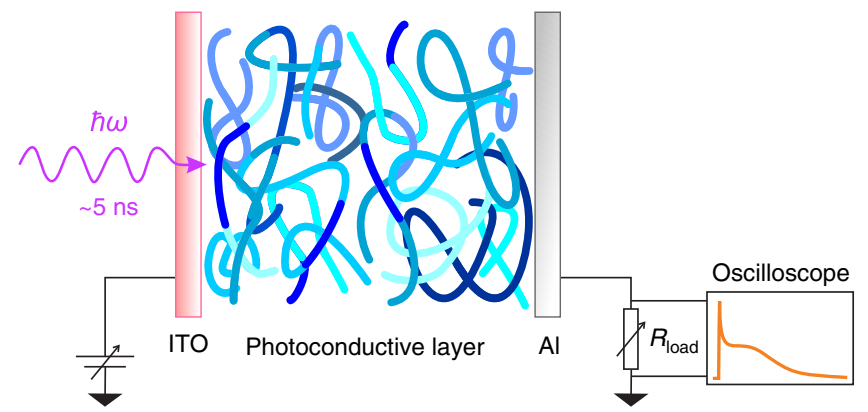

FIG. 16. Simplified schematic of the time-of-flight (TOF) photocurrent setup with a zoom-in of the TOF device structure. Concerning the photoconductive layer of the device, the varied blue shades across the spatially disordered polymer chains symbolize the presence of energetic disorder among chargetransport sites. For all relevant studies presented in this work, TOF devices were prepared by having ITO as the front ("transparent") electrode and aluminium as the counter electrode. Figure components are not illustrated to scale.

metal enclosure (fitted with a fused-silica optical window to allow for optical excitation) for (i) sample preservation against degradation and (ii) electromagnetic interference rejection.

Temperature-dependent (cryogenic) TOF measurements were carried out using an OptistatDN liquid nitrogen cryostat (Oxford Instruments) adapted with miniature $50 \Omega$ coaxial cables (RG178B/U; Lapp Kabel) for electrical signaling.

\section{Reconstruction of HDR TOF photocurrent transients}

Concerning TOF post-transit photocurrent analysis, the logarithmic dependence of energy on time implies that acquisition of HDR photocurrent with a large time span is essential for estimating the profile of localized states lying deep in the bandgap. As for the passive resistive sensing approach, it relies on a terminating variable resistor to convert current transients into time-varying voltage signals proportional to the choice of sensing resistance. In order to obtain transients with extended time and dynamic range, one can progressively assign increasing values of sensing resistance by varying a resistor wired in parallel with the $1-\mathrm{M} \Omega$ termination of the scope. It can be expected that applying higher resistance is advantageous for probing deeper into the post-transit regime due to higher measurement sensitivity and better SNR. However, an enlarged $R C$ time constant of the setup would, meanwhile, suppress the ability to capture high-frequency features that a photocurrent carries, typically following its initial onset. By carefully balancing the interplay between measurement sensitivity and the speed of system response, and overlaying ("stitching" together) individual photocurrent sections obtained from incremented resistor values, we are able to reconstruct a single piece of TOF photocurrent transient with extended dynamic range. We employ a 
commercially available variable resistor (VT1, Thorlabs) for our passive-mode HDR photocurrent measurements. The VT1 unit features a feedthrough configuration to the BNC inputs of the scope. Having the variable resistor set $(50,100,500,1 \mathrm{k}, 5 \mathrm{k}, 10 \mathrm{k}$, and $50 \mathrm{k}$ ohms $)$ fully integrated within its miniaturized metal housing, this unit provides a superior electromagnetic shielding. When necessary, further higher resistance values can be applied using fixed stub-style terminators FT104 $(100 \mathrm{k} \Omega)$ and FT254 $(250 \mathrm{k} \Omega$ ) (both from Thorlabs) paired with a BNC T-connector. Along with adjusting the sensing resistances, we also exploit the finite horizontal (500 points in fast sampling mode) and vertical (9-bit, i.e., 512 points) resolutions of the digital scope, as we note that acquisition of photocurrent sections by tuning "volts/div" and "time/ div" settings properly for a given sensing resistance could also benefit the reconstruction of a whole HDR transient.

Figure 17 provides an example of such reconstructed HDR photocurrent transients, where its constituent sections are distinguished by multicolors, with each color corresponding to a value of sense resistor chosen to collect the data. Spanning circa 7 orders of magnitude in current levels and 6 orders of magnitude in time, this pieced HDR transient preserves both the early timescale features (sub100-ns region) and the signal fidelity in the long-time limit.

\section{Current density-voltage measurements on single-carrier devices}

A Keithley SMU 236 automated via a LabVIEW computer interface was used to record the $J-V$ response

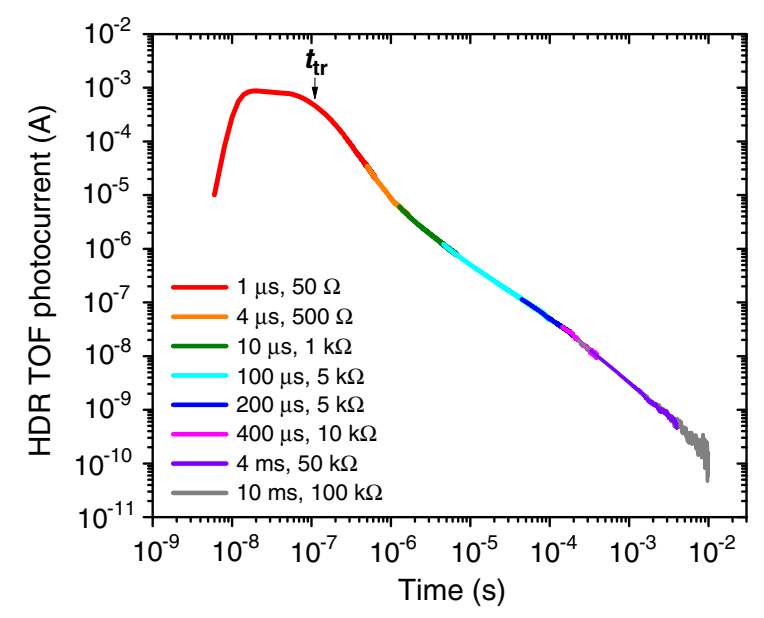

FIG. 17. An example of HDR TOF photocurrent transients. It is reconstructed by overlaying a set of transient sections captured with successively incremented sensing resistances (see legend for details). The device under test (DUT), a glassy TOF device of about $1.8 \mu \mathrm{m}$ made from HMW PFO, was probed at room temperature under $10-\mathrm{V}$ external bias and with a fixed laser excitation intensity (such that $Q / Q_{0} \approx 15 \%$ ). Pre- and posttransit regimes of the HDR transient are distinguished by an arrow denoting the carrier transit time $\left(t_{\mathrm{tr}}\right)$. of a series of single-carrier devices of structure ITO/PEDOT:PSS/PFO/ $\mathrm{MoO}_{x} / \mathrm{Au}$, with the PFO active layers prepared in either fully glassy or $\beta$-phase embedded states. The typical voltage scan step is 20 or $25 \mathrm{mV}$. The devices were kept in the dark and inert $\mathrm{N}_{2}$ atmosphere during measurements.

\section{Mobility determination from frequency-dependent imaginary part of the impedance}

Frequency-resolved probes were further applied to single-carrier devices, in order to extract charge-carrier mobilities and compare degrees of transport dispersion for a given device configuration with either the presence or absence of the molecular conformational traps. The hole-only devices are assumed as locally linear, causal, and time-invariant (LTI) systems. A frequency-variable small-signal ac perturbation (50 $\mathrm{mV}_{p p}$ to ensure local linearity) was applied on top of a set of discrete dc bias voltages during the measurements. The variable dc bias dictates the "background" spatial charge-carrier density and electrostatic profiles across a device.

A portable electrochemical impedance analyzer CompactStat (Ivium Technologies B.V.) was used with measurement interface IviUMSOFT software v2.454. Impedance spectroscopies on asymmetric hole-only devices were performed based on a two-electrode setup, that is, having the counter electrode (CE) and the reference electrode (RE) wired onto the blocking device contact, while the working electrode (WE) and the sense terminal (S) joint are connected with the opposite contact capable of providing Ohmic injection of carriers.

The steady-state (dc) charge-carrier mobility $\mu_{\mathrm{e} / \mathrm{h}}$ can be determined from the $-\operatorname{Im}[Z]-f$ data as [37]

$$
\mu_{\mathrm{e} / \mathrm{h}}=\frac{d^{2} f_{\text {peak }}}{\gamma V_{\mathrm{dc}}}
$$

where $d$ is the active layer thickness, $V_{\mathrm{dc}}$ a set of discrete dc bias voltages that tracks along the steady-state $J-V$ curve [114], $f_{\text {peak }}$ the peak frequency of each $-\operatorname{Im}[Z]$ versus $\log _{10}(f)$ spectrum at a given dc bias, and $\gamma$ a numerical factor that relates the time constant $\tau_{c}$ (i.e., $1 / f_{\text {peak }}$ ) from the frequency-resolved $-\operatorname{Im}[Z]$ data [115] to the dc transit time $\left(\tau_{\mathrm{dc}}\right)$ of carriers. The value of $\gamma$ is not clearly determined in the literature $[34,37,78,116]$ and has been identified as a variable depending on the degree of transport dispersion [37], typically ranging from about 0.35 to 0.6. By recognizing that the actual selection of this prefactor $\gamma$ value will by no means significantly affect the magnitude of estimated mobility, we have chosen $\gamma=0.5$ for all measurement scenarios where the $-\operatorname{Im}[Z]-f$ method was applied.

Additional to the aforementioned $-\operatorname{Im}[Z]-f$ method for steady-state carrier mobility extraction, we have also exploited a negative differential susceptance $(-\Delta B-f)$ 
method $[34,78,116,117]$ to characterize and directly compare transport dispersion among hole-only devices nominally prepared from the same material, however treated via varied processing routes. The determination of carrier mobility using the $-\Delta B-f$ method relies on the ability to locate the characteristic concave "dips" in the $C-f$ spectra, a phenomenon that only occurs in low-dispersive transport. However, the degree of transport-hence the applicability of the method-can be mediated by the inclusion or exclusion (abbreviated as " + /-") of certain sub-bandgap trap states relating to specific polymorph(s) of a given material system. Such an example is demonstrated in Figs. S11-S14 of the SM [72], where hole transport in LMW $+/-\beta$ hole-only devices is concerned. When the $-\Delta B-f$ method was applied for mobility extraction, we adopted $\gamma=0.56$, as proposed by Tsang et al. for disordered molecular semiconductors with low carrier dispersion [34].

All impedance spectroscopic data were analyzed using ZVIEW software v3.4d.

\section{Energy-resolved electrochemical impedance spectroscopy (ER-EIS)}

This recently developed method is capable of mapping the DOS in organic semiconductors over wide energy ranges [81,82]. The ER-EIS method is based on the interaction between the organic film and electrolyte via reduction-oxidation (redox) reactions. This interaction, which is similar to the capture-extraction process of an electron with the DOS in organic semiconductors, results in a recombination current $j_{\text {rec }}$. The desired DOS function of electronic transport and defect states, $g(E)$, can be derived directly from the measured charge-transfer resistance of a semiconductor-electrolyte interface, $R_{\mathrm{CT}}$, at the position of the Fermi energy $E_{\mathrm{F}}$ and at a frequency where the redox reactions determine the real part of the impedance [82]:

$$
\left[R_{\mathrm{CT}}\left(E_{\mathrm{F}}\right)\right]^{-1}=S \frac{\partial j_{\mathrm{rec}}}{\partial V}=q^{2} k_{\mathrm{ET}} S[A] g\left(E_{\mathrm{F}}\right),
$$

where $q$ is the elementary charge, $k_{\mathrm{ET}}$ the charge-transfer rate constant (assumed energy-independent), $S$ the sample area, and $[A]$ the concentration of the electrolyte redox species in the interphase region of the solid-liquid contact.

The DOS function $g(E)$ in a semiconductor at the electrochemical potential, $E_{\mathrm{F}}=q V_{\mathrm{app}}$, can be expressed in terms of $R_{\mathrm{CT}}$ measured at the given applied dc voltage $\left(V_{\text {app }}\right)$ as $[81,82]$

$$
g\left(E_{\mathrm{F}}\right)=\left(q^{2} k_{\mathrm{ET}} S[A] R_{\mathrm{CT}}\right)^{-1} .
$$

The $R_{\mathrm{CT}}$ is experimentally measured by the harmonic perturbation $d V$ with a proper frequency. Such small-signal amplitude $d V$ superimposed on an external dc bias $V_{\text {app }}$ thus provides direct information about the electronic DOS at the energy adjusted by varying $V_{\text {app }}$.

A Solartron Analytical 1260 impedance/gain-phase analyzer was used for the ER-EIS experiments. The frequency was set to $0.5 \mathrm{~Hz}$, the amplitude of ac voltage was $100 \mathrm{mV}$, and the average scan rate of the dc voltage ramp was $10 \mathrm{mV} \mathrm{s}^{-1}$. The measurements were performed in a glove box with protective $\mathrm{N}_{2}$ atmosphere (with oxygen and moisture below $20 \mathrm{ppm}$ and $2 \mathrm{ppm}$, respectively) using a common three-electrode electrochemical cell with volume of about $200 \mu \mathrm{L}$ and a working electrode area of about $12 \mathrm{~mm}^{2}$. Solution of $0.1-\mathrm{M} \mathrm{TBAPF}{ }_{6}$ in acetonitrile was used as the supporting electrolyte. The potential of the working electrode with respect to the reference $\mathrm{Ag} / \mathrm{AgCl}$ electrode was controlled via a home-built potentiostat. Pt wire was used as the counter electrode. The energy recorded relative to the reference $\mathrm{Ag} / \mathrm{AgCl}$ electrode can be referred to the local vacuum level given that the $\mathrm{Ag} / \mathrm{AgCl}$ energy departs from the vacuum value by $4.66 \mathrm{eV}$.

\section{APPENDIX D: SPECTROSCOPIC SIGNATURES OF GLASSY AND $\beta$-PHASE EMBEDDED PFO FILMS}

We examine photophysical properties of prepared PFO films to confirm that the chosen processing protocols indeed yield the required solid-state microstructures. UV-Vis absorption spectra were recorded using a dual-beam Shimadzu UV-2600 spectrophotometer equipped with a diffuse reflectivity (integrating sphere) attachment, which allows the spectra to be corrected for reflection and scattering losses. Photoluminescence (PL) spectra were recorded using a Jobin Yvon Horiba FluoroMax-3 spectrofluorometer in reflection geometry (with an angle of incidence of about $75^{\circ}$ to minimize reabsorption effect). The PL excitation was chosen at $385 \mathrm{~nm}$.

Figure 18(a) shows the obtained UV-Vis absorption spectra. Glassy PFO films exhibit a typical, inhomogeneously broadened absorption spectrum (peaking at about $381 \mathrm{~nm}$ ) that arises from a stochastic distribution of intermonomer torsion angles for the ensemble of kinetically trapped, nonequilibrium chain conformations obtained following hot spin coating [79] (and hence a broad distribution of electronic coupling between adjacent polymer repeat units). The absorption spectrum for $\beta$-phase embedded PFO films displays the characteristic redshifted peak at about $432 \mathrm{~nm}$, attributed to the $S_{0} \rightarrow S_{1} 0-0$ transition for the extended, planar-zigzag-type $\beta$-phase chain segments $[65,118]$. The $\beta$-phase absorption peak is especially well defined because (i) $\beta$-phase is understood to form with a certain minimum segment length [92], and (ii) the $\beta$-phase formation process - namely, co-crystallization with a small-molecular solvent [119]—inherently requires 

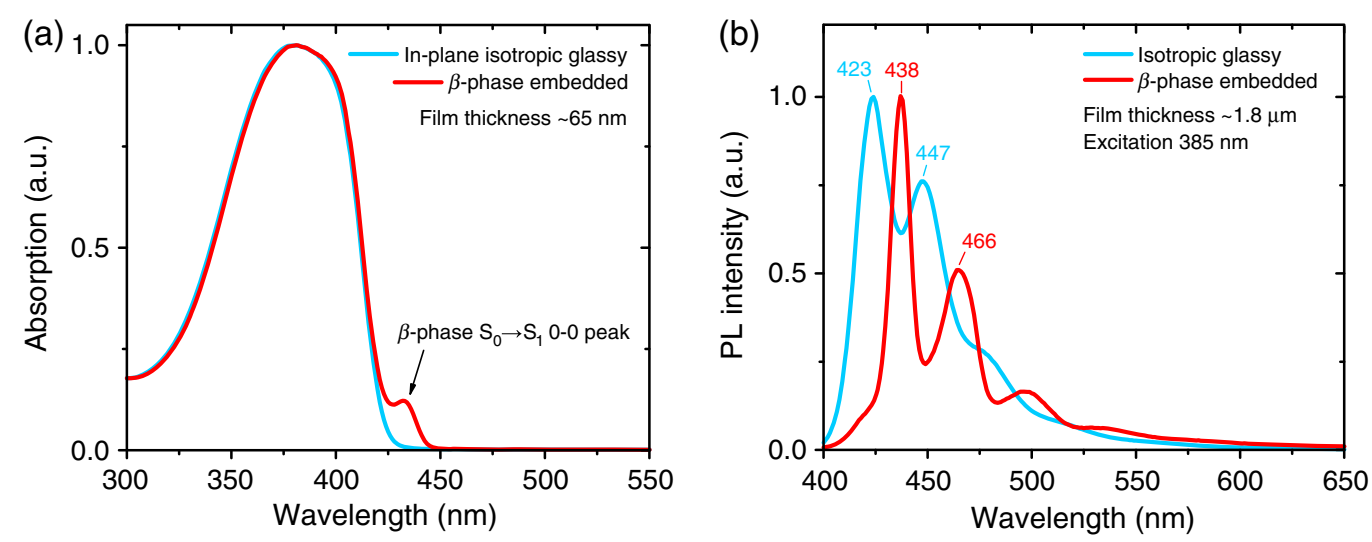

FIG. 18. Optical spectroscopy of HMW PFO films of two distinct microstructures due to varied processing: isotropic glassy (blue line) via spin coating at elevated temperature, $\beta$-phase embedded (red line) via toluene solvent vapor exposure to pristine glassy films. (a) Peak-normalized absorption spectra for thin $(\sim 65 \mathrm{~nm})$ films. (b) Fluorescent emission from glassy and $\beta$-phase embedded optically thick $(\sim 1.8 \mu \mathrm{m})$ PFO layers that were used in TOF measurements. Signature wavelengths of the emission peaks in both cases are labeled. Note in the $\beta$-phase embedded PL a 420-nm glassy $\mathrm{S}_{1} \rightarrow \mathrm{S}_{0} 0-0$ shoulder. The PL spectra were recorded with excitation at $385 \mathrm{~nm}$.

a distinct molecular geometry in terms of both the backbone and the side-chain arrangements [120,121].

Figure 18(b) shows PL spectra of the $+/-\beta$ TOF films normalized to their respective $S_{1} \rightarrow S_{0} 0-0$ vibronic peaks.

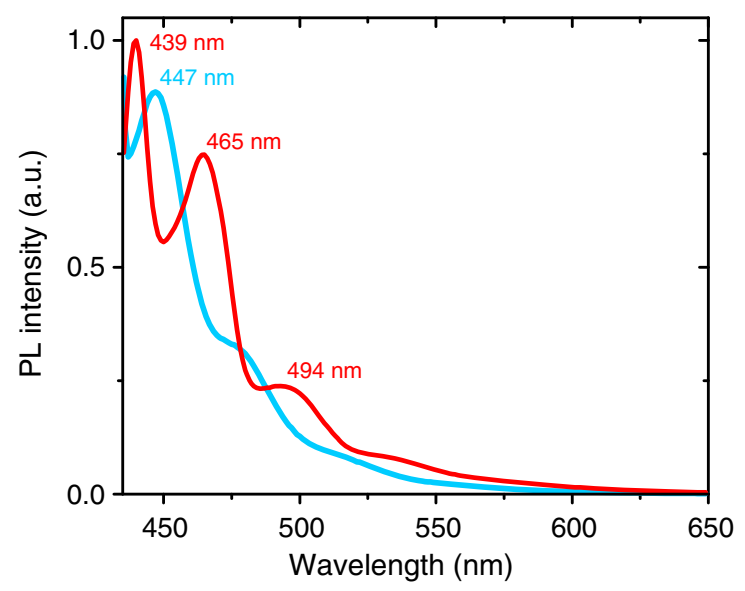

FIG. 19. PL spectra of thick $(\sim 1.8 \mu \mathrm{m})$ glassy (blue line) and $\beta$-phase (red line) PFO films, as used for TOF measurements, recorded with excitation at $432 \mathrm{~nm}$. This excitation wavelength closely matches the $\mathrm{S}_{0}-\mathrm{S}_{1} 0-0$ absorption peak of the $\beta$-phase (see Fig. 18) and will therefore select sites containing $\beta$-phase segments. Such site-selective excitation populates any $\beta$-phase chain segments directly, in contrast to excitation at shorter wavelengths that would populate $\beta$-phase segments via exciton energy transfer. This method is thus a more sensitive spectroscopic probe of the presence of $\beta$-phase, and the absence of emission characteristic of $\beta$-phase for the glassy film confirms that the glassy PFO films are indeed maximally free from $\beta$-phase. The wavelengths corresponding to the vibronic peaks are indicated, showing excellent agreement with the ones in Fig. 18(b), and with previously published spectroscopic data for glassy and $\beta$-phase PFO $[65,113,123,124]$.
The spectra emphasize the (i) redshifts, (ii) sharpened linewidths, and (iii) reduced apparent Huang-Rhys factors [122] of the vibronic transitions upon planarization of backbone (glassy $\rightarrow \beta$-phase), which in turn promotes intrachain electronic coupling and delocalization of the excited state. Note that for $\beta$-phase embedded films, due to efficient excitation energy transfer, the PL emission originates almost entirely from those minority chainextended species, with only a slight shoulder at about $420 \mathrm{~nm}$ signifying the $S_{1} \rightarrow S_{0} 0-0$ transition peak of glassy-state PFO.

Furthermore, site-selective PL measurements confirmed that the glassy PFO films are maximally free from either crystalline or $\beta$-phase chain segments (see Fig. 19 and caption for details) — an important feature for our charge transport study.

\section{APPENDIX E: ESTIMATING VOLUME FRACTION OF INDUCED $\beta$-PHASE BY RAMAN SCATTERING}

Figure 20 illustrates the approach we took to estimating the volume fraction (vol\%) of the $\beta$-phase present in the $\beta$-phase embedded TOF layers by quantifying the relative intensity of a Raman mode that features a characteristic dependence on the $\beta$-phase fraction [125]. Note that for these thick layers, conventional analysis of the $\beta$-phase fraction based on optical transmission measurements (as applicable to thin films) is problematic due to their excessively large optical depth.

Previously reported Raman spectroscopy measurements on PFO films (see Ref. [67]) have revealed a linear relation between the relative intensity of selected Raman peaks and the $\beta$-phase fraction, albeit for PFO of somewhat lower molecular weight. Notably, the Raman intensity ratio $r_{R}$ of 


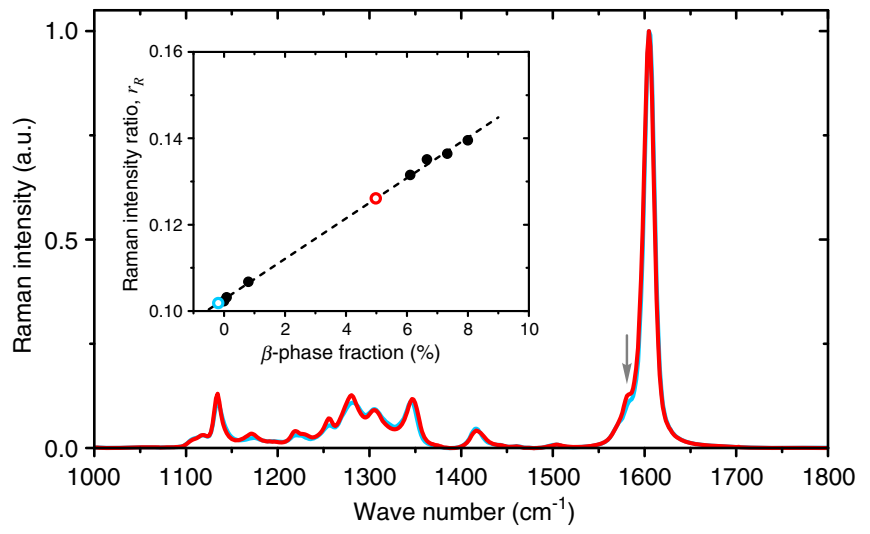

FIG. 20. Estimation of $\beta$-phase vol\% in HMW TOF (i.e., optically thick) layers using glassy (blue line) and $\beta$-phase embedded (red line) Raman spectra. A grey arrow indicates the spectral position of the $1581 \mathrm{~cm}^{-1}$ mode. The inset shows the previously reported [67] experimental data and linear fit (filled circle and dashed line, respectively) for the Raman intensity ratio $r_{R}$ of the $1581 \mathrm{~cm}^{-1}$ mode relative to the $1606 \mathrm{~cm}^{-1}$ mode as a function of the $\beta$-phase fraction. The corresponding $r_{R}$ values extracted from the Raman spectra of glassy (blue open circle) and $\beta$-phase embedded (red open circle) TOF PFO films are placed on the fit line to provide estimates for their respective $\beta$-phase fractions.

the $1581-\mathrm{cm}^{-1}$ mode relative to the $1606-\mathrm{cm}^{-1}$ mode [126] was reported to scale linearly with the $\beta$-phase fraction varying in the range of $0-9 \mathrm{vol} \%$. This linear relation reads

$r_{R}=4.68 \times 10^{-3} \times[\beta$-phase fraction $(\%)]+0.103$

While the generality of this relation in terms of, for instance, molecular weight of PFO is not explicitly determined to date, it was nevertheless deemed appropriate for estimating the $\beta$-phase fraction in thick PFO layers used in TOF devices.

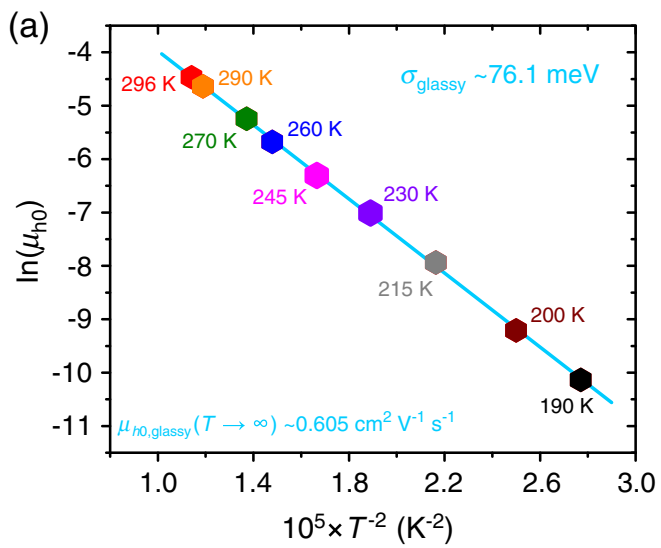

To this end, Raman spectra were recorded for glassy and $\beta$-phase embedded TOF films using instrumental settings identical to those in Ref. [67] and are shown in Fig. 20. The corresponding Raman intensity ratios $r_{R}$ of the $1581-\mathrm{cm}^{-1}$ mode relative to the $1606-\mathrm{cm}^{-1}$ mode were then placed on the previously reported fit line in order to assess the $\beta$-phase fraction (see inset of Fig. 20).

For the $\beta$-phase embedded HMW PFO TOF film, a value of about 0.126 was found for $r_{R}$; this analysis yields an estimate of $\sim 4.6 \pm 0.4 \%$ for the $\beta$-phase fraction. Gratifyingly, for the glassy PFO TOF film $\left(r_{R}=0.102\right)$, the $\beta$-phase fraction is estimated to be -0.2 , which closely matches the nominal $0 \%$ value.

We estimated, in the same manner, about $6 \pm 0.1 \%$ $\beta$-phase content for LMW layers subjected to the controlled $1 \mathrm{hrSVE}$ treatment (see Fig. 14 for processing details).

\section{APPENDIX F: ENERGETIC-DISORDER BREADTH ANALYSIS BASED ON THE GAUSSIAN DISORDER MODEL}

It is a fairly common practice in the literature on disordered low-mobility organic photoconductive media to extract disorder parameters within the framework of the GDM [6]. The GDM assumes the DOS profiles to be of Gaussian type with standard deviation (aka breadth) $\sigma_{\mathrm{GDM}}$. The analysis would allow us to compare results with previously published data on similar (e.g., polyfluorenetype) materials. In addition, although the GDM formalism is not directly consistent with our device model (as presented in Sec. IV, Part B), it serves to evaluate freehole mobility in the case of glassy devices that feature active layers with microstructural homogeneity and isotropy.

The GDM analysis as shown in Fig. 21 yields a slightly higher value for the energetic disorder of the $\beta$-phase embedded device $\left(\sigma_{\mathrm{GDM}, \beta \text {-embedded }} \approx 85 \mathrm{meV}\right)$ than for the

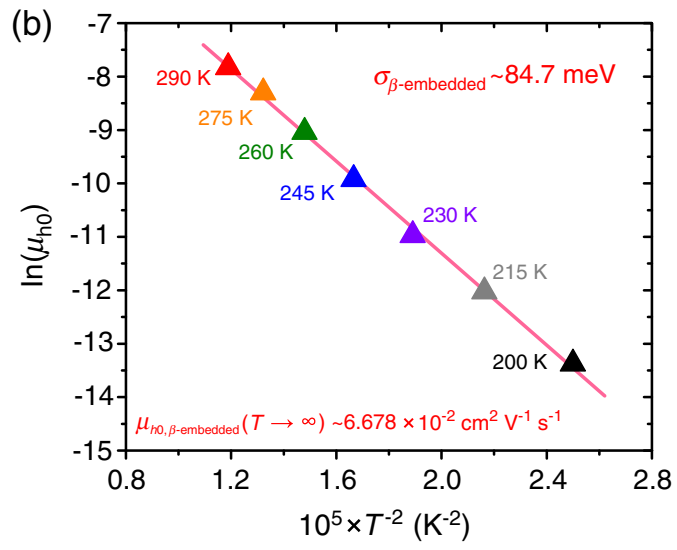

FIG. 21. Analysis of temperature-dependent HMW PFO TOF hole mobility data based on the GDM. The plots show zero-field hole mobility $\mu_{h 0}$ versus $T^{-2}$ for the estimation of energetic disorder breadth $\sigma$ and high-temperature-limit $\mu_{h 0}$, based on GDM fitting of the experimental TOF $\mu_{h}$ data as in Fig. 2(b): (a) for the glassy device and (b) for the $\beta$-phase embedded device. 
neat glassy device $\left(\sigma_{\mathrm{GDM} \text {,glassy }} \approx 75 \mathrm{meV}\right)$. A broadened disorder breadth for the $\beta$-phase embedded PFO would be expected, due to the presence of conformational deep trap states. However, it should be stressed that for the above analysis, the Gaussian form of DOS is a significant simplification to the realistic DOS distributions in these materials, and therefore the derived values of $\sigma$ should be treated with reservation. It also provides a high-temperature limit of $\mu_{h 0}\left(\mu_{h 0(T \rightarrow \infty)}\right)$ for the glassy device of about $0.6 \mathrm{~cm}^{2} \mathrm{~V}^{-1} \mathrm{~s}^{-1}$, which we assign as the effective "free" hole mobility (or the band edge $\mu_{h}$ ) above the HOMO mobility edge in the framework of the multiple-trapping model. We believe $\mu_{h 0(T \rightarrow \infty)}$ could provide a guide to the magnitude of hole mobility in a scenario that always features abundant thermal activation to populate carriers up in the mobile band. Although we recognize that the physical significance of the effective free charge mobility from the GDM is not clear, it was simply used as a guide to the magnitude of free-hole mobility of the mobile band through the amorphous PFO medium. This value-which is of order of $1 \mathrm{~cm}^{2} \mathrm{~V}^{-1} \mathrm{~s}^{-1}$-is compatible with timeresolved-microwave-conductivity-assessed intrachain mobility along coiled PFO chains in dilute solution $[52,73]$. Traditionally, the estimate of $\mu_{h 0}$ in the hightemperature limit has been correlated with the degree of electronic coupling (aka overlap integral) between transport sites for material systems comparison. Here, we argue that this is not always applicable, especially for active layers bearing structural heterogeneities (e.g., in the case of $\beta$-phase embedded PFO).

\section{APPENDIX G: IMPEDANCE SPECTROSCOPIES OF PFO HOLE-ONLY DEVICES WITH OR WITHOUT $\beta$-PHASE}

Impedance spectroscopies on the HMW PFO hole-only devices with and without the inclusion $(+/-)$ of the $\beta$-phase were performed using a frequency-variable $(100-\mathrm{Hz}$ to $1-2-\mathrm{MHz}) 50-\mathrm{mV}_{p p}$ small-signal ac perturbation at discrete forward dc biases. The (arguably simplistic) equivalent circuit applied to model the system behavior is provided as an inset of Fig. 22, along with example Nyquist plots of a series of low-bias data sets probed from the $\beta$-embedded device, is shown in the main figure. The complete experimental data, arranged in the form of the negative imaginary part of the impedance versus frequency $(-\operatorname{Im}[Z]-f)$, on both the $+/-\beta$ devices, are presented in Fig. 23.

The circuit model, a simplified Randles circuit $[127,128]$ (Fig. 22 inset) is chosen since it yields well-fit semicircles as a function of forward dc bias (Fig. 22). This choice allows us to evaluate hole-only device parameters such as series resistance $R_{s}$, geometric capacitance $C_{\text {geo }}$, etc. In reality, the equivalent circuit would surely be more sophisticated than the simplified Randles treatment since, for instance,

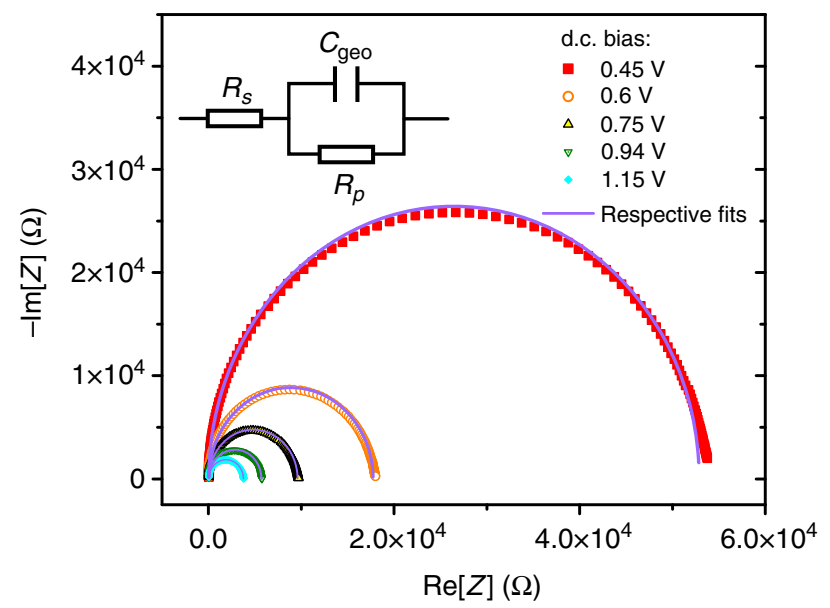

FIG. 22. "Unconstrained" (see surrounding text for clarifications) semicircle fits to the low-voltage data series of the $135-\mathrm{nm}$ HMW PFO $\beta$-embedded hole-only device, using the simplified Randles equivalent circuit (schematic provided as the figure inset).

the charge-trapping effect is not taken into account by such a circuit model, nor does the model include any semiempirical circuit elements such as constant phase elements (CPE) to account for nonideal capacitive behavior originating from, e.g., dispersive carrier transport. However, we note that often "less is more": From these unconstrained fits-obtained without restricting any of the three components in the equivalent circuit to produce an individual optimal semicircle fit to the impedance data set at each dc bias-we found $R_{s} \sim 50-100 \Omega$ (useful later for hole-only device modeling), $C_{\mathrm{geo}} \sim 830-870 \mathrm{pF}$ [129]. In addition, as expected from the superlinear forward-bias $J-V$ response [as shown in Fig. 3(a)], the overall conductivity across the device rockets (as $R_{p}$ rapidly drops) with increased voltage bias.

\section{Normalized $-\operatorname{Im}(Z)$-frequency response}

Figure 23 plots, side by side, the acquired $+/-\beta$ impedance spectroscopy data in the normalized $-\operatorname{Im}[Z]$ versus $\log _{10}(f)$ presentation. By performing numerical simulation of $C-f$ and $-\operatorname{Im}[Z]-f$ characteristics that takes into account various degrees of transport dispersion, Tripathi et al. demonstrated that the $-\operatorname{Im}[Z]-f$ method is resilient even to the presence of high dispersive transport; hence, it affords reliable determination of dc mobility [37]. In addition, the relative degree of dispersion can be compared by inspecting the relative breadth of the $-\operatorname{Im}[Z]$ spectra among different systems, with a narrower $-\operatorname{Im}[Z]-f$ distribution corresponding to a lower dispersion. Concerning Fig. 23, no discernible difference in either the FWHM or the overall shape of the $+/-\beta$ normalized $-\operatorname{Im}[Z]$ spectra is presentwe note that this observation is gratifyingly consistent with the similar transport dispersions $\left(W_{\text {glassy }} \approx W_{\beta \text {-embedded }} \approx\right.$ 

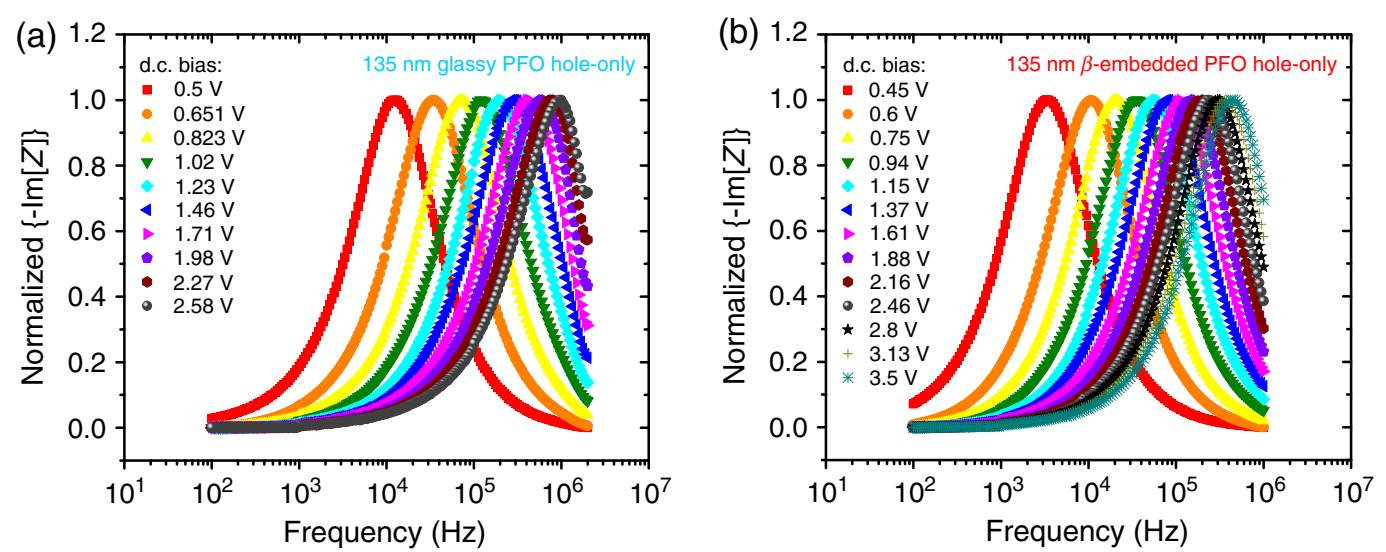

FIG. 23. Normalized frequency-dependent - $\operatorname{Im}[Z]$ spectra of 135-nm asymmetric HMW PFO hole-only devices in (a) glassy and (b) $\beta$-embedded states. At each given dc bias, we reciprocate the frequency peak of the $-\operatorname{Im}[Z]$ spectrum to a dc hole transit time, from which a corresponding steady-state hole mobility can be calculated [as per Eq. (C1)]. The estimated $\mu_{h}$ set for each device awaits further correction for the built-in voltage $V_{\mathrm{bi}}$ in order to finalize the evaluation of field-dependent steady-state $\mu_{h}$, as shown in Fig. 25 .

0.25) [130] extracted from TOF photocurrent transients, as shown in Fig. 2(a).

We therefore apply Eq. (C1) based on the $-\operatorname{Im}[Z]-f$ method [37] to extract field-dependent steady-state (dc) hole mobility $\left(\mu_{h}\right)$ for $+/-\beta$ hole-only devices.

\section{Capacitance-voltage characteristics}

There remains an additional requirement for correcting the dc biases for the built-in voltage $V_{\mathrm{bi}}$ of the asymmetric hole-only devices in order to work out their respective fielddependent dc mobility values. To this end, we turn to capacitance-voltage $(C-V)$ measurements $[38,131]$. The experimental $C-V$ characteristics are provided in Fig. 24.

We identify, from Fig. 24(b), the occurrence of a (first) characteristic capacitive peak around the $V_{\text {bi }}$ region under forward bias. This peak is associated with the competition between the drift and diffusion current components near the $\mathrm{MoO}_{x} / \mathrm{PFO}$ interface: The diffusion term causes an increase [132] in charge-carrier density when the dc bias closely approaches $V_{\mathrm{bi}}$, while the drift term sweeps away the

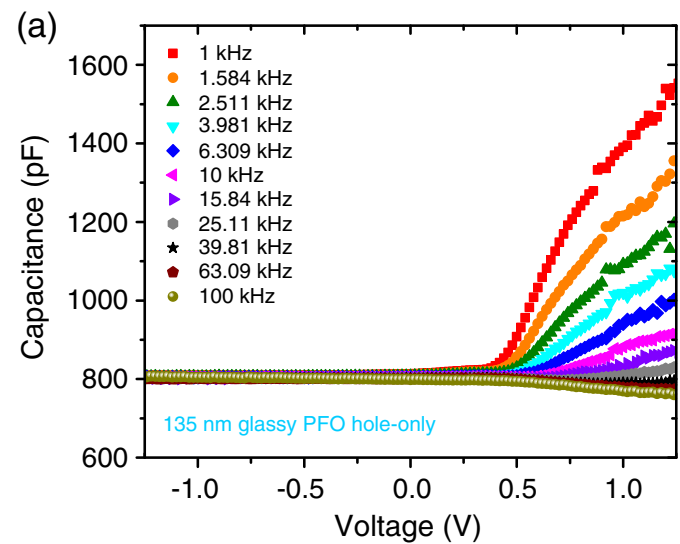

accumulated carriers thereby reducing the capacitance $[38,131,133]$. At further increased forward-bias voltages, the hole current motion across the device proceeds towards the drift-dominant, space-charge-limited regime, while the capacitance rolls off towards three-quarters (0.75) of the geometric capacitance $C_{\text {geo }}[38,131,134]$. Moreover, the frequency-dependent capacitance response of semiconductor diodes in the presence of localized defect states $[22,40,135,136]$ has been well documented in the literature. In conjunction with probing the intrinsic $C-V$ caused by the gradual transition from diffusion- to drift-governed transport, because of the frequency-tunable small-signal ac modulation, the $C-V$ measurements inherently capture an extra capacitance contribution from charges thermally released from trap states. We present the underlying reasons as follows.

Given the reciprocity between time and frequency, we recall from Eq. (2) that the maximum frequency, at which the trapped carriers are still able to interact with the ac modulation and thus contribute to the junction capacitance,

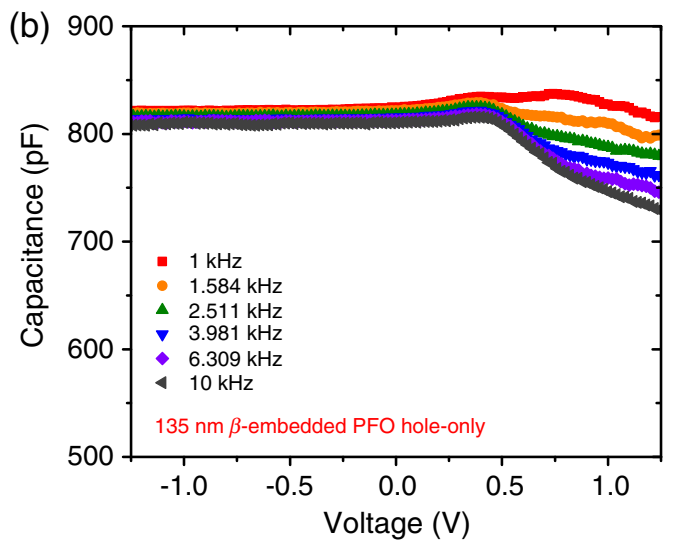

FIG. 24. Frequency-dependent $C-V$ characteristics of (a) glassy and (b) $\beta$-embedded 135-nm asymmetric HMW PFO hole-only devices. Positive voltage means applying positive potentials to the $\mathrm{Au} / \mathrm{MoO}_{x}$ contact relative to the ITO/PEDOT:PSS end. 

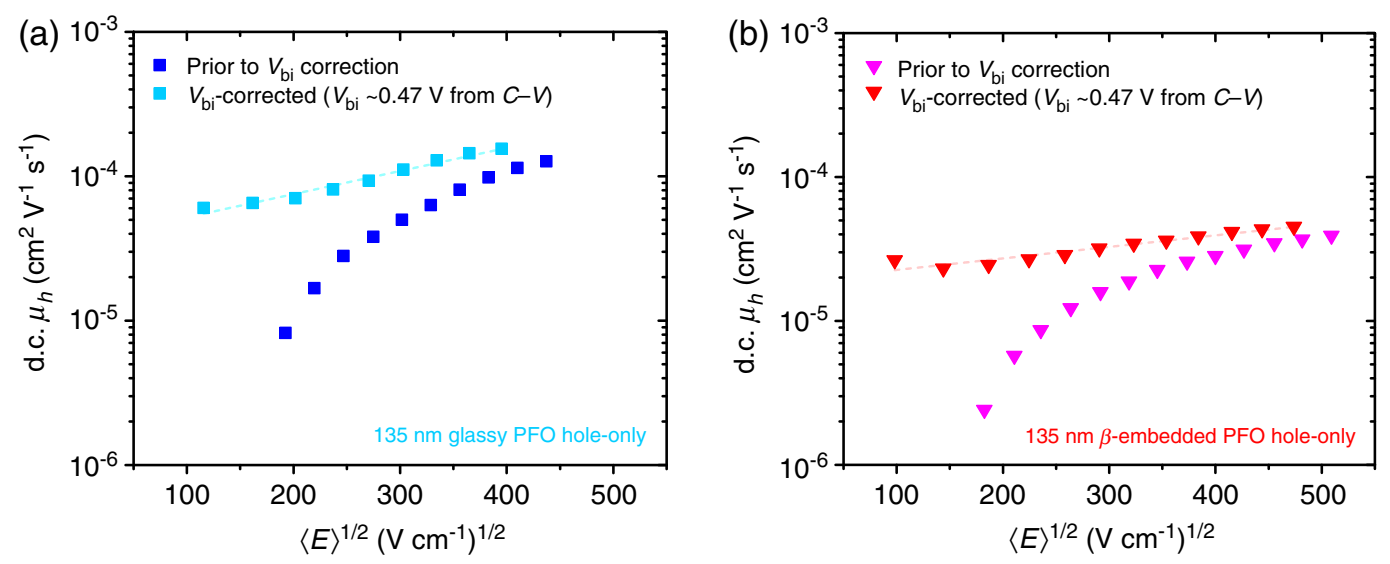

FIG. 25. The $V_{\mathrm{bi}}$-corrected field-dependent dc hole mobilities for (a) glassy and (b) $\beta$-embedded 135-nm HMW PFO hole-only devices [the higher mobility sets in both subfigures (a) and (b); same as shown in Fig. 4]. The subfigures also retain de $\mu_{h}$ data prior to built-in voltage correction (the two lower mobility sets).

is given by the trap-depth-dependent carrier emission rate [137]. The $C-V$ spectra probed via a frequency-variable ac perturbation (1-100 kHz for the present measurement as in Fig. 24) therefore reflect the landscape of sub-bandgap DOS by modulating the occupancy of the defect states (or ODOS): A higher-frequency small signal interacts with trap levels in closer vicinity to the HOMO transport level, and it interrogates further into the sub-bandgap space as its frequency decreases [138].

As for the glassy hole-only device [shown in Fig. 24(a)], the capacitance in the forward-bias regime is more pronouncedly affected by the aforementioned charge-trapping effect [139], hence hampering the assessment of $V_{\mathrm{bi}}$. Despite the different trap-mediated forward-bias capacitance response between $+/-\beta$ devices, under reverse bias where hole injection is effectively blocked, the devices otherwise signify the geometric capacitance [134], with $C_{\text {geo }}$ for both devices of about $810 \mathrm{pF}$ : This implies an identical thickness of the two device variants and agrees with surface profilometry measurements.

Following the above rationale, we extract, from Fig. 24(b), $V_{\mathrm{bi}}$ of $\beta$-embedded hole-only devices by locating the potential at which the capacitance value in the forward-bias regime equals the geometric capacitance $C_{\text {geo }}$ inferred from the reverse-bias regime [131], provided that the value is no longer a function of further increased frequency of the $50-\mathrm{mV}_{p p}$ small-signal ac perturbation (i.e., from, namely, either the $6.309-\mathrm{kHz}$ or $10-\mathrm{kHz}$ trace). The $V_{\mathrm{bi}}$ is thus estimated to be about $0.47 \mathrm{~V}$.

Figure 25 summarizes the estimated dc $\mu_{h}$ prior to and after accounting for $V_{\mathrm{bi}}$.

[1] N. F. Mott, E. A. Davis, and R. A. Street, States in the Gap and Recombination in Amorphous Semiconductors, Philos. Mag. 32, 961 (1975).
[2] T. Tiedje and A. Rose, A Physical Interpretation of Dispersive Transport in Disordered Semiconductors, Solid State Commun. 37, 49 (1981).

[3] J. Orenstein and M. Kastner, Photocurrent Transient Spectroscopy: Measurement of the Density of Localized States in a-As2Se3, Phys. Rev. Lett. 46, 1421 (1981).

[4] G. F. Seynhaeve, R. P. Barclay, G. J. Adriaenssens, and J. M. Marshall, Post-Transit Time-of-Flight Currents as a Probe of the Density of States in Hydrogenated Amorphous Silicon, Phys. Rev. B 39, 10196 (1989).

[5] K. Koughia, Z. Shakoor, S. O. Kasap, and J. M. Marshall, Density of Localized Electronic States in a-Se from Electron Time-of-Flight Photocurrent Measurements, J. Appl. Phys. 97, 033706 (2005).

[6] H. Bässler, Charge Transport in Disordered Organic Photoconductors: A Monte Carlo Simulation Study, Phys. Status Solidi B 175, 15 (1993).

[7] S. L. M. van Mensfoort, J. Billen, S. I. E. Vulto, R. A. J. Janssen, and R. Coehoorn, Electron Transport in Polyfluorene-Based Sandwich-Type Devices: Quantitative Analysis of the Effects of Disorder and Electron Traps, Phys. Rev. B 80, 033202 (2009).

[8] J. Rivnay, R. Noriega, J. E. Northrup, R. J. Kline, M. F. Toney, and A. Salleo, Structural Origin of Gap States in Semicrystalline Polymers and the Implications for Charge Transport, Phys. Rev. B 83, 121306 (2011).

[9] R. A. Street, Localized State Distribution and Its Effect on Recombination in Organic Solar Cells, Phys. Rev. B 84, 075208 (2011).

[10] T. Kirchartz, B. E. Pieters, J. Kirkpatrick, U. Rau, and J. Nelson, Recombination via Tail States in Polythiophene: fullerene Solar Cells, Phys. Rev. B 83, 115209 (2011).

[11] R. C. I. MacKenzie, C. G. Shuttle, M. L. Chabinyc, and J. Nelson, Extracting Microscopic Device Parameters from Transient Photocurrent Measurements of P3HT:PCBM Solar Cells, Adv. Energy Mater. 2, 662 (2012).

[12] W. S. C. Roelofs, S. G. J. Mathijssen, R. A. J. Janssen, D. M. de Leeuw, and M. Kemerink, Accurate Description of Charge Transport in Organic Field Effect Transistors 
Using an Experimentally Extracted Density of States, Phys. Rev. B 85, 085202 (2012).

[13] S. Mehraeen, V. Coropceanu, and J.-L. Brédas, Role of Band States and Trap States in the Electrical Properties of Organic Semiconductors: Hopping Versus Mobility Edge Model, Phys. Rev. B 87, 195209 (2013).

[14] M. K. Fung, S. L. Lai, S. N. Bao, C. S. Lee, J. J. O’Briein, M. Inbasekaran, W. W. Wu, and S. T. Lee, Photoelectron Spectroscopic Studies of Poly(9,9-dioctylfluorene)Potassium Interface, and Its Influence by Oxygen, Synth. Met. 128, 97 (2002).

[15] X. Lin, G. E. Purdum, Y. Zhang, S. Barlow, S. R. Marder, Y.-L. Loo, and A. Kahn, Impact of a Low Concentration of Dopants on the Distribution of Gap States in a Molecular Semiconductor, Chem. Mater. 28, 2677 (2016).

[16] W. Zhang, J. Smith, S. E. Watkins, R. Gysel, M. McGehee, A. Salleo, J. Kirkpatrick, S. Ashraf, T. Anthopoulos, M. Heeney, and I. McCulloch, Indacenodithiophene Semiconducting Polymers for High-Performance, Air-Stable Transistors, J. Am. Chem. Soc. 132, 11437 (2010).

[17] Y. Tsutsui et al., Unraveling Unprecedented Charge Carrier Mobility through Structure Property Relationship of Four Isomers of Didodecyl[1]benzothieno[3,2-b] [1]benzothiophene, Adv. Mater. 28, 7106 (2016).

[18] A. J. Kronemeijer, V. Pecunia, D. Venkateshvaran, M. Nikolka, A. Sadhanala, J. Moriarty, M. Szumilo, and H. Sirringhaus, Two-Dimensional Carrier Distribution in Top-Gate Polymer Field-Effect Transistors: Correlation between Width of Density of Localized States and Urbach Energy, Adv. Mater. 26, 728 (2014).

[19] D. Venkateshvaran, M. Nikolka, A. Sadhanala, V. Lemaur, M. Zelazny, M. Kepa, M. Hurhangee, A. J. Kronemeijer, V. Pecunia, I. Nasrallah, I. Romanov, K. Broch, I. McCulloch, D. Emin, Y. Olivier, J. Cornil, D. Beljonne, and H. Sirringhaus, Approaching Disorder-Free Transport in High-Mobility Conjugated Polymers, Nature (London) 515, 384 (2014).

[20] P. Stallinga, H. L. Gomes, H. Rost, A. B. Holmes, M. G. Harrison, and R.H. Friend, Electronic Levels in MEH-PPV, Synth. Met. 111, 535 (2000).

[21] A. J. Campbell, D. D. C. Bradley, E. Werner, and W. Brütting, Deep Level Transient Spectroscopy (DLTS) of a Poly(p-phenylene vinylene) Schottky Diode, Synth. Met. 111, 273 (2000).

[22] P. P. Boix, G. Garcia-Belmonte, U. Muñecas, M. Neophytou, C. Waldauf, and R. Pacios, Determination of Gap Defect States in Organic Bulk Heterojunction Solar Cells from Capacitance Measurements, Appl. Phys. Lett. 95, 233302 (2009).

[23] B. Ecker, J. C. Nolasco, J. Pallarés, L. F. Marsal, J. Posdorfer, J. Parisi, and E. von Hauff, Degradation Effects Related to the Hole Transport Layer in Organic Solar Cells, Adv. Funct. Mater. 21, 2705 (2011).

[24] J. A. Carr, M. Elshobaki, and S. Chaudhary, Deep Defects and the Attempt to Escape Frequency in Organic Photovoltaic Materials, Appl. Phys. Lett. 107, 203302 (2015).

[25] U. K. Verma, D. C. Tripathi, and Y. N. Mohapatra, Direct Determination of Defect Density of States in Organic Bulk Heterojunction Solar Cells, Appl. Phys. Lett. 109, 113301 (2016).
[26] T. Dylla, S. Reynolds, R. Carius, and F. Finger, Electron and Hole Transport in Microcrystalline Silicon Solar Cells Studied by Time-of-Flight Photocurrent Spectroscopy, J. Non. Cryst. Solids 352, 1093 (2006).

[27] C. G. Shuttle, N. D. Treat, J. D. Douglas, J. M. J. Fréchet, and M. L. Chabinyc, Deep Energetic Trap States in Organic Photovoltaic Devices, Adv. Energy Mater. 2, 111 (2012).

[28] R. H. Bube, Photoconductivity of Solids (Wiley and Sons, New York, 1960).

[29] C. Renaud, C. H. Huang, C. W. Lee, P. Le Rendu, and T. P. Nguyen, Study of Trap States in Polyfluorene Based Devices by Using TSC Technique, Thin Solid Films 516, 7209 (2008).

[30] J. Schafferhans, A. Baumann, C. Deibel, and V. Dyakonov, Trap Distribution and the Impact of Oxygen-Induced Traps on the Charge Transport in Poly(3-hexylthiophene), Appl. Phys. Lett. 93, 093303 (2008).

[31] J. Schafferhans, C. Deibel, and V. Dyakonov, Electronic Trap States in Methanofullerenes, Adv. Energy Mater. 1, 655 (2011).

[32] A. Kadashchuk, A. Vakhnin, Y. Skryshevski, V. I. Arkhipov, E. V. Emelianova, and H. Bässler, Thermally Stimulated Luminescence in $\pi$-conjugated Polymers Containing Fluorene and Spirobifluorene Units, Chem. Phys. 291, 243 (2003).

[33] A. Kadashchuk, R. Schmechel, H. von Seggern, U. Scherf, and A. Vakhnin, Charge-Carrier Trapping in Polyfluorene-Type Conjugated Polymers, J. Appl. Phys. 98, 024101 (2005).

[34] S. W. Tsang, S. K. So, and J. B. Xu, Application of Admittance Spectroscopy to Evaluate Carrier Mobility in Organic Charge Transport Materials, J. Appl. Phys. 99, 013706 (2006).

[35] G. Garcia-Belmonte, P. P. Boix, J. Bisquert, M. Sessolo, and H. J. Bolink, Simultaneous Determination of Carrier Lifetime and Electron Density-of-States in P3HT:PCBM Organic Solar Cells under Illumination by Impedance Spectroscopy, Sol. Energy Mater. Sol. Cells 94, 366 (2010).

[36] J. M. Montero and J. Bisquert, Interpretation of TrapLimited Mobility in Space-Charge Limited Current in Organic Layers with Exponential Density of Traps, J. Appl. Phys. 110, 043705 (2011).

[37] D. C. Tripathi, A. K. Tripathi, and Y. N. Mohapatra, Mobility Determination Using Frequency Dependence of Imaginary Part of Impedance (Im Z) for Organic and Polymeric Thin Films, Appl. Phys. Lett. 98, 033304 (2011).

[38] D. C. Tripathi and Y. N. Mohapatra, Diffusive Capacitance in Space Charge Limited Organic Diodes: Analysis of Peak in Capacitance-Voltage Characteristics, Appl. Phys. Lett. 102, 253303 (2013).

[39] J. Bisquert, L. Bertoluzzi, I. Mora-Sero, and G. GarciaBelmonte, Theory of Impedance and Capacitance Spectroscopy of Solar Cells with Dielectric Relaxation, Drift-Diffusion Transport, and Recombination, J. Phys. Chem. C 118, 18983 (2014).

[40] L. Xu, J. Wang, and J. W. P. Hsu, Transport Effects on Capacitance-Frequency Analysis for Defect Characterization in Organic Photovoltaic Devices, Phys. Rev. Applied 6, 064020 (2016). 
[41] V. Coropceanu, J. Cornil, D. A. da Silva Filho, Y. Olivier, R. Silbey, and J.-L. Brédas, Charge Transport in Organic Semiconductors, Chem. Rev. 107, 926 (2007).

[42] B. Baumeier, J. Kirkpatrick, and D. Andrienko, DensityFunctional Based Determination of Intermolecular Charge Transfer Properties for Large-Scale Morphologies, Phys. Chem. Chem. Phys. 12, 11103 (2010).

[43] C. Poelking and D. Andrienko, Long-Range Embedding of Molecular Ions and Excitations in a Polarizable Molecular Environment, J. Chem. Theory Comput. 12, 4516 (2016).

[44] C. Groves, Simulating Charge Transport in Organic Semiconductors and Devices: A Review, Reports Prog. Phys. 80, 026502 (2017).

[45] S. V. Novikov, Hopping Charge Transport in Amorphous Semiconductors with the Spatially Correlated Exponential Density of States, J. Chem. Phys. 146, 024504 (2017).

[46] Z. Bao, A. Dodabalapur, and A. J. Lovinger, Soluble and Processable Regioregular Poly(3-hexylthiophene) for Thin Film Field-Effect Transistor Applications with High Mobility, Appl. Phys. Lett. 69, 4108 (1996).

[47] P. Prins, F. C. Grozema, J. M. Schins, S. Patil, U. Scherf, and L. D. A. Siebbeles, High Intrachain Hole Mobility on Molecular Wires of Ladder-Type Poly(p-Phenylenes), Phys. Rev. Lett. 96, 146601 (2006).

[48] P. Prins, F. C. Grozema, B. S. Nehls, T. Farrell, U. Scherf, and L. D. A. Siebbeles, Enhanced Charge-Carrier Mobility in $\beta$-phase Polyfluorene, Phys. Rev. B 74, 113203 (2006).

[49] W. Zhang, J. Smith, R. Hamilton, M. Heeney, J. Kirkpatrick, K. Song, S. E. Watkins, T. Anthopoulos, and I. McCulloch, Systematic Improvement in Charge Carrier Mobility of Air Stable Triarylamine Copolymers, J. Am. Chem. Soc. 131, 10814 (2009).

[50] H. Huang, Z. Chen, R. P. Ortiz, C. Newman, H. Usta, S. Lou, J. Youn, Y.-Y. Noh, K.-J. Baeg, L. X. Chen, A. Facchetti, and T. Marks, Combining Electron-Neutral Building Blocks with Intramolecular "Conformational Locks" Affords Stable, High-Mobility P-and N-Channel Polymer Semiconductors, J. Am. Chem. Soc. 134, 10966 (2012).

[51] E. Giussani, D. Fazzi, L. Brambilla, M. Caironi, and C. Castiglioni, Molecular Level Investigation of the Film Structure of a High Electron Mobility Copolymer via Vibrational Spectroscopy, Macromolecules 46, 2658 (2013).

[52] M. J. Bird, O. G. Reid, A. R. Cook, S. Asaoka, Y. Shibano, H. Imahori, G. Rumbles, and J. R. Miller, Mobility of Holes in Oligo- and Polyfluorenes of Defined Lengths, J. Phys. Chem. C 118, 6100 (2014).

[53] D. P. McMahon, D. L. Cheung, L. Goris, J. Dacuña, A. Salleo, and A. Troisi, Relation between Microstructure and Charge Transport in Polymers of Different Regioregularity, J. Phys. Chem. C 115, 19386 (2011).

[54] R. Noriega, A. Salleo, and A. J. Spakowitz, Chain Conformations Dictate Multiscale Charge Transport Phenomena in Disordered Semiconducting Polymers, Proc. Natl. Acad. Sci. U.S.A. 110, 16315 (2013).

[55] C. Poelking and D. Andrienko, Effect of Polymorphism, Regioregularity and Paracrystallinity on Charge
Transport in Poly(3-hexylthiophene) [P3HT] Nanofibers, Macromolecules 46, 8941 (2013).

[56] J. M. Frost, J. Kirkpatrick, T. Kirchartz, and J. Nelson, Parameter Free Calculation of the Subgap Density of States in Poly(3-hexylthiophene), Faraday Discuss. 174, 255 (2014).

[57] P. Gemünden, C. Poelking, K. Kremer, K. Daoulas, and D. Andrienko, Effect of Mesoscale Ordering on the Density of States of Polymeric Semiconductors, Macromol. Rapid Commun. 36, 1047 (2015).

[58] A. Troisi and A. Shaw, Very Large $\pi$-Conjugation Despite Strong Nonplanarity: A Path for Designing New Semiconducting Polymers, J. Phys. Chem. Lett. 7, 4689 (2016).

[59] S. A. Mollinger, A. Salleo, and A. J. Spakowitz, Anomalous Charge Transport in Conjugated Polymers Reveals Underlying Mechanisms of Trapping and Percolation, ACS Cent. Sci. 2, 910 (2016).

[60] D. Hertel, U. Scherf, and H. Bässler, Charge Carrier Mobility in a Ladder-Type Conjugated Polymer, Adv. Mater. 10, 1119 (1998).

[61] D. D. C. Bradley, M. Grell, X. Long, H. Mellor, A. W. Grice, M. Inbasekaran, and E.P. Woo, Influence of Aggregation on the Optical Properties of a Polyfluorene, in Proceedings of the Optical Science, Engineering and Instrumentation '97, San Diego, CA, USA, 1997, Vol. 3145 (1997), p. 254, https://doi.org/10.1117/12.295530.

[62] M. Grell, D. D. C. Bradley, X. Long, T. Chamberlain, M. Inbasekaran, E. P. Woo, and M. Soliman, Chain Geometry, Solution Aggregation and Enhanced Dichroism in the Liquidcrystalline Conjugated Polymer Poly(9,9dioctylfluorene), Acta Polym. 49, 439 (1998).

[63] M. Grell, D. D. C. Bradley, G. Ungar, J. Hill, and K. S. Whitehead, Interplay of Physical Structure and Photophysics for a Liquidcrystalline Polyfluorene, Macromolecules 32, 5810 (1999).

[64] M. E. Caruso and M. Anni, Real-Time Investigation of Solvent Swelling Induced $\beta$-phase Formation in Poly (9,9-dioctylfluorene), Phys. Rev. B 76, 054207 (2007).

[65] A. Perevedentsev, Y. Sonnefraud, C. R. Belton, S. Sharma, A. E. G. Cass, S. A. Maier, J.-S. Kim, P. N. Stavrinou, and D. D. C. Bradley, Dip-Pen Patterning of Poly(9,9-dioctylfluorene) Chain-Conformation-Based Nano-photonic Elements, Nat. Commun. 6, 5977 (2015).

[66] W. C. Tsoi and D. G. Lidzey, Raman Spectroscopy of Fluorene Oligomers in the $\alpha$-, $\beta$ - and $\gamma$-phases, J. Phys. Condens. Matter 20, 125213 (2008).

[67] A. Perevedentsev, N. Chander, J.-S. Kim, and D. D. C. Bradley, Spectroscopic Properties of Poly(9,9dioctylfluorene) Thin Films Possessing Varied Fractions of $\beta$-phase Chain Segments: Enhanced Photoluminescence Efficiency via Conformation Structuring, J. Polym. Sci. Part B Polym. Phys. 54, 1995 (2016).

[68] M. Redecker and D. D. C. Bradley, Mobility Enhancement through Homogeneous Nematic Alignment of a LiquidCrystalline Polyfluorene, Appl. Phys. Lett. 74, 1400 (1999).

[69] T. Kreouzis, D. Poplavskyy, S. M. Tuladhar, M. CampoyQuiles, J. Nelson, A. J. Campbell, and D. D. C. Bradley, Temperature and Field Dependence of Hole Mobility in 
Poly(9,9-dioctylfluorene), Phys. Rev. B 73, 235201 (2006).

[70] S. Foster, Ph.D. Thesis, Imperial College London, United Kingdom, 2013.

[71] X. Shi, Ph.D. Thesis, Imperial College London, United Kingdom, 2018.

[72] See Supplemental Material at http://link.aps.org/ supplemental/10.1103/PhysRevX.9.021038 for "Relating Chain Conformation and the Density of States to Charge Transport in Conjugated Polymers".

[73] P. Prins, F. C. Grozema, F. Galbrecht, U. Scherf, and L. D. A. Siebbeles, Charge Transport along Coiled Conjugated Polymer Chains, J. Phys. Chem. C 111, 11104 (2007).

[74] H. T. Nicolai, G. A. H. Wetzelaer, M. Kuik, A. J. Kronemeijer, B. de Boer, and P.W. M. Blom, SpaceCharge-Limited Hole Current in Poly(9,9-dioctylfluorene) Diodes, Appl. Phys. Lett. 96, 172107 (2010).

[75] J. A. Röhr, X. Shi, S. A. Haque, T. Kirchartz, and J. Nelson, Charge Transport in Spiro-OMeTAD Investigated through Space-Charge-Limited Current Measurements, Phys. Rev. Applied 9, 044017 (2018).

[76] J. A. Röhr, D. Moia, S. A. Haque, T. Kirchartz, and J. Nelson, Exploring the Validity and Limitations of the Mott-Gurney Law for Charge-Carrier Mobility Determination of Semiconducting Thin-Films, J. Phys. Condens. Matter 30, 105901 (2018).

[77] N. F. Mott and R. W. Gurney, Electronic Processes in Ionic Crystals, 2/e (Dover, New York, 1964).

[78] H. C. F. Martens, J. N. Huiberts, and P. W. M. Blom, Simultaneous Measurement of Electron and Hole Mobilities in Polymer Light-Emitting Diodes, Appl. Phys. Lett. 77, 1852 (2000).

[79] A. Perevedentsev, S. Aksel, K. Feldman, P. Smith, P. N. Stavrinou, and D. D. C. Bradley, Interplay between Solid State Microstructure and Photophysics for Poly(9,9dioctylfluorene) within Oriented Polyethylene Hosts, J. Polym. Sci. Part B Polym. Phys. 53, 22 (2015).

[80] R. C. I. Mackenzie, C. G. Shuttle, G. F. Dibb, N. Treat, E. von Hauff, M. J. Robb, C. J. Hawker, M. L. Chabinyc, and J. Nelson, Interpreting the Density of States Extracted from Organic Solar Cells Using Transient Photocurrent Measurements, J. Phys. Chem. C 117, 12407 (2013).

[81] V. Nádaždy, F. Schauer, and K. Gmucová, Energy Resolved Electrochemical Impedance Spectroscopy for Electronic Structure Mapping in Organic Semiconductors, Appl. Phys. Lett. 105, 142109 (2014).

[82] F. Schauer, V. Nádaždy, and K. Gmucová, Electrochemical Impedance Spectroscopy for Study of Electronic Structure in Disordered Organic Semiconductors-Possibilities and Limitations, J. Appl. Phys. 123, 161590 (2018).

[83] K. Gmucová, V. Nádaždy, F. Schauer, M. Kaiser, and E. Majková, Electrochemical Spectroscopic Methods for the Fine Band Gap Electronic Structure Mapping in Organic Semiconductors, J. Phys. Chem. C 119, 15926 (2015).

[84] V. Nádaždy, K. Gmucová, P. Nádaždy, P. Siffalovic, K. Vegso, M. Jergel, F. Schauer, and E. Majkova, Thickness Effect on Structural Defect-Related Density of States and Crystallinity in P3HT Thin Films on ITO Substrates, J. Phys. Chem. C 122, 5881 (2018).
[85] F. Schauer, M. Tkáčová, V. Nadáždy, K. Gmucová, M. Ožvoldová, L. Tkáč, and J. Chlpík, Electronic Structure of UV Degradation Defects in Polysilanes Studied by Energy Resolved-Electrochemical Impedance Spectroscopy, Polymer degradation and stability 126, 204 (2016).

[86] S. Volk, N. Yazdani, E. Sanusoglu, O. Yarema, M. Yarema, and V. Wood, Measuring the Electronic Structure of Nanocrystal Thin Films Using Energy-Resolved Electrochemical Impedance Spectroscopy, J. Phys. Chem. Lett. 9, 1384 (2018).

[87] L. S. Liao, L. F. Cheng, M. K. Fung, C. S. Lee, S. T. Lee, M. Inbasekaran, E. P. Woo, and W. W. Wu, Interface Formation between Poly(9,9-dioctylfluorene) and Ca Electrode Investigated Using Photoelectron Spectroscopy, Chem. Phys. Lett. 325, 405 (2000).

[88] S. Janietz, D. D. C. Bradley, M. Grell, C. Giebeler, M. Inbasekaran, and E. P. Woo, Electrochemical Determination of the Ionization Potential and Electron Affinity of Poly(9,9-dioctylfluorene), Appl. Phys. Lett. 73, 2453 (1998).

[89] C. Tanase, E. J. Meijer, P. W. M. Blom, and D. M. de Leeuw, Unification of the Hole Transport in Polymeric Field-Effect Transistors and Light-Emitting Diodes, Phys. Rev. Lett. 91, 216601 (2003).

[90] N. Vukmirović and L.-W. Wang, Density of States and Wave Function Localization in Disordered Conjugated Polymers: A Large Scale Computational Study, J. Phys. Chem. B 115, 1792 (2011).

[91] E. M. Rice, Ph.D. Thesis, Imperial College London, United Kingdom, 2018.

[92] W. C. Tsoi, A. Charas, A. J. Cadby, G. Khalil, A. M. Adawi, A. Iraqi, B. Hunt, J. Morgado, and D. G. Lidzey, Observation of the $\beta$-Phase in Two Short-Chain Oligofluorenes, Adv. Funct. Mater. 18, 600 (2008).

[93] The 1D drift-diffusion model is available at https://www .gpvdm.com.

[94] L. Sims, U. Hörmann, R. Hanfland, R. C. I. MacKenzie, F. R. Kogler, R. Steim, W. Brütting, and P. Schilinsky, Investigation of the S-Shape Caused by the Hole Selective Layer in Bulk Heterojunction Solar Cells, Org. Electron. 15, 2862 (2014).

[95] N. Felekidis, A. Melianas, and M. Kemerink, Nonequilibrium Drift-Diffusion Model for Organic Semiconductor Devices, Phys. Rev. B 94, 035205 (2016).

[96] R. C. I. MacKenzie, V. S. Balderrama, S. Schmeisser, R. Stoof, S. Greedy, J. Pallarès, L. F. Marsal, A. Chanaewa, and E. von Hauff, Loss Mechanisms in High Efficiency Polymer Solar Cells, Adv. Energy Mater. 6, 1501742 (2016).

[97] R. C. I. MacKenzie, A. Göritz, S. Greedy, E. von Hauff, and J. Nelson, Theory of Stark spectroscopy transients from thin film organic semiconducting devices, Phys. Rev. B 89, 195307 (2014).

[98] A. Melianas, F. Etzold, T. J. Savenije, F. Laquai, O. Inganäs, and M. Kemerink, Photo-Generated Carriers Lose Energy During Extraction from Polymer-Fullerene Solar Cells, Nat. Commun. 6, 8778 (2015).

[99] O. Tal, Y. Rosenwaks, Y. Preezant, N. Tessler, C. K. Chan, and A. Kahn, Direct Determination of the Hole Density of States in Undoped and Doped Amorphous Organic Films 
with High Lateral Resolution, Phys. Rev. Lett. 95, 256405 (2005).

[100] W. L. Kalb, S. Haas, C. Krellner, T. Mathis, and B. Batlogg, Trap Density of States in Small-Molecule Organic Semiconductors: A Quantitative Comparison of Thin-Film Transistors with Single Crystals, Phys. Rev. B 81, 155315 (2010).

[101] J. Dacuña and A. Salleo, Modeling Space-Charge-Limited Currents in Organic Semiconductors: Extracting Trap Density and Mobility, Phys. Rev. B 84, 195209 (2011).

[102] L. Huang, X. Huang, G. Sun, C. Gu, D. Lu, and Y. Ma, Study of $\beta$ Phase and Chains Aggregation Degrees in Poly (9,9-dioctylfluorene) (PFO) Solution, J. Phys. Chem. C 116, 7993 (2012).

[103] A. Salleo, Charge Transport in Polymeric Transistors, Mater. Today 10, 38 (2007).

[104] M. Redecker, D. D. C. Bradley, M. Inbasekaran, and E. P. Woo, Nondispersive Hole Transport in an Electroluminescent Polyfluorene, Appl. Phys. Lett. 73, 1565 (1998).

[105] A. J. Cadby, P. A. Lane, H. Mellor, S. J. Martin, M. Grell, C. Giebeler, D. D. C. Bradley, M. Wohlgenannt, C. An, and Z. V. Vardeny, Film Morphology and Photophysics of Polyfluorene, Phys. Rev. B 62, 15604 (2000).

[106] M. Abkowtz and D. M. Pai, Comparison of the Drift Mobility Measured under Transient and Steady-State Conditions in a Prototypical Hopping System, Philos. Mag. B 53, 193 (1986).

[107] K. K. H. Chan, S. W. Tsang, H. K. H. Lee, F. So, and S. K. So, Charge Injection and Transport Studies of Poly(2,7carbazole) Copolymer PCDTBT and Their Relationship to Solar Cell Performance, Org. Electron. 13, 850 (2012).

[108] K. H. Cheon, J. Cho, B. T. Lim, H.-J. Yun, S.-K. Kwon, Y.-H. Kim, and D. S. Chung, Analysis of Charge Transport in High-Mobility Diketopyrrolopyrole Polymers by Space Charge Limited Current and Time of Flight Methods, RSC Adv. 4, 35344 (2014).

[109] R. A. Marcus, On the Theory of Oxidation-Reduction Reactions Involving Electron Transfer. I, J. Chem. Phys. 24, 966 (1956).

[110] B. K. Yap, R. Xia, M. Campoy-Quiles, P. N. Stavrinou, and D. D. C. Bradley, Simultaneous Optimization of ChargeCarrier Mobility and Optical Gain in Semiconducting Polymer Films, Nat. Mater. 7, 376 (2008).

[111] P. E. Parris, V. M. Kenkre, and D. H. Dunlap, Nature of Charge Carriers in Disordered Molecular Solids: Are Polarons Compatible with Observations?, Phys. Rev. Lett. 87, 126601 (2001).

[112] Using Chromafil® PTFE filters (Macherey-Nagel GmbH $\&$ Co. KG) of 1 - or $0.45-\mu \mathrm{m}$ pore sizes for HMW and LMW materials, respectively.

[113] S. H. Chen, A. G. Su, C. H. Su, and S. A. Chen, Crystalline Forms and Emission Behavior of Poly(9,9-di-n-octyl-2,7fluorene), Macromolecules 38, 379 (2005).

[114] Thereby granting this method the inherent capability of extracting field-dependent carrier mobility.

[115] The $f_{\text {peak }}$ equivalently manifests at the "top" of the (near) semicircles in a Nyquist plot (e.g., Fig. 22).

[116] K. K. H. Chan, S. W. Tsang, H. K. H. Lee, F. So, and S. K. So, Charge Transport Study of Semiconducting Polymers and Their Bulk Heterojunction Blends by Capacitance Measurements, J. Polym. Sci. Part B Polym. Phys. 51, 649 (2013).

[117] With $-\Delta B(f)=-f\left(C-C_{\text {geo }}\right)$, where $C_{\text {geo }}$ is the geometric capacitance of the device.

[118] D. L. Huber and I. Avgin, Optical Absorption of the $\beta$ Phase of Poly(9,9-dioctylfluorene), J. Polym. Sci. Part B Polym. Phys. 54, 1109 (2016).

[119] A. Perevedentsev, P. N. Stavrinou, P. Smith, and D. D. C. Bradley, Solution-Crystallization and Related Phenomena in 9,9-dialkyl-fluorene Polymers. II. Influence of SideChain Structure, J. Polym. Sci. Part B Polym. Phys. 53, 1492 (2015).

[120] W. Chunwaschirasiri, B. Tanto, D. L. Huber, and M. J. Winokur, Chain Conformations and Photoluminescence of Poly(di-n-octylfluorene), Phys. Rev. Lett. 94, 107402 (2005).

[121] C. Volz, M. Arif, and S. Guha, Conformations in Dioctyl Substituted Polyfluorene: A Combined Theoretical and Experimental Raman Scattering Study, J. Chem. Phys. 126, 064905 (2007).

[122] In other words, the increase of the recorded ratios of $\mathrm{S}_{1} \rightarrow \mathrm{S}_{0}$ 0-0 vibronic transition relative to the 0-1 and 0-2.

[123] M. Ariu, D. G. Lidzey, M. Sims, A. J. Cadby, P. A. Lane, and D. D. C. Bradley, The Effect of Morphology on the Temperature-Dependent Photoluminescence Quantum Efficiency of the Conjugated Polymer Poly(9,9dioctylfluorene), J. Phys. Condens. Matter 14, 9975 (2002).

[124] S. H. Chen, A. C. Su, and S. A. Chen, Noncrystalline Phases in Poly(9,9-di-n-octyl-2,7-fluorene), J. Phys. Chem. B 109, 10067 (2005).

[125] This characteristic dependence is largely attributable to the mode's sensitivity to enhanced electronic polarizability arising from the planarized $\beta$-phase chain segments.

[126] A main peak of about $1606 \mathrm{~cm}^{-1}$ is assigned to a symmetric in-plane $\mathrm{C}-\mathrm{C}$ ring-stretching mode [92].

[127] J. E. B. Randles, Kinetics of Rapid Electrode Reactions, Discuss. Faraday Soc. 1, 11 (1947).

[128] The Warburg diffusion element $Z_{w}$ is omitted in such simplified Randles circuit, as we expect a drift-dominated behavior within the devices under the given measurement settings, including (i) small-signal frequency $\geq 100 \mathrm{~Hz}$ and (ii) the lowest applied dc bias voltages $\geq V_{\mathrm{bi}}$ (the reader is referred to the incoming $C-V$ measurement results for the estimation of $V_{\mathrm{bi}}$ ).

[129] Similar to $C_{\text {geo }}$ values inferred from $C-f$ measurements, vide infra (Fig. 24).

[130] Commonly read as $W=\left(t_{1 / 2}-t_{\text {tr }}\right) / t_{1 / 2}$, where $t_{1 / 2}$ is the time at which the current decreases to half of its plateau value [6].

[131] S. L. M. van Mensfoort and R. Coehoorn, Determination of Injection Barriers in Organic Semiconductor Devices from Capacitance Measurements, Phys. Rev. Lett. 100, 086802 (2008).

[132] Primarily due to a reduction in local band bending close to the contact.

[133] D. C. Tripathi and Y. N. Mohapatra, Charge Transport across Organic Heterostructure: Role of Interfacial Density of States, J. Appl. Phys. 116, 064509 (2014). 
[134] J. Shao and G. T. Wright, Characteristics of the SpaceCharge-Limited Dielectric Diode at Very High Frequencies, Solid State Electron. 3, 291 (1961).

[135] E. P. EerNisse, Accurate Capacitance Calculations for PN Junctions Containing Traps, Appl. Phys. Lett. 18, 183 (1971).

[136] L. C. Kimerling, Influence of Deep Traps on the Measurement of Free-Carrier Distributions in Semiconductors by Junction Capacitance Techniques, J. Appl. Phys. 45, 1839 (1974).

[137] Note that $R_{\text {escape }}=\nu_{0} e^{-\left(E / k_{\mathrm{B}} T\right)}$; hence, the deepest trap level still capable of responding to an ac small signal with angular frequency $\omega=2 \pi f$ is given by $E^{*}(\omega)=$ $k_{\mathrm{B}} T \ln \left(\nu_{0} \omega^{-1}\right)$.

[138] Therefore, greater capacitance contributions from defects at lower modulation frequencies, as in Fig. 24.

[139] In the case of a glassy device, the conspicuous trapmediated characteristics arise from not having an energetically deep-lying and distinct distribution of localized sub-bandgap states, as in the case of the $\beta$-embedded device. Alternatively, to intrinsically circumvent from the trapping effect, high-quality diodes realized by nearly trapfree active layers (made from, e.g., conjugated small molecules via vapor deposition) would be required [38]. 